\title{
Multiplicative Consistency Ascertaining, Inconsistency Repairing, and Weights Derivation of Hesitant Multiplicative Preference Relations
}

\author{
Yejun $\mathrm{Xu}^{\left({ }^{(}\right)}$, Mengqi Li, Francisco Chiclana ${ }^{(}$, , and Enrique Herrera-Viedma ${ }^{\circledR}$, Fellow, IEEE
}

\begin{abstract}
This article investigates multiplicative consistency ascertaining, inconsistency repairing, and weights derivation for hesitant multiplicative preference relations (HMPRs). First, the completely multiplicative consistency and weakly multiplicative consistency of HMPRs are defined. Based on them, 0-1 mixed programming models and simple algebraic operations are proposed to ascertain the multiplicative consistency of HMPRs. Then, some goal programming models are developed to generate the weights from consistent HMPRs and to revise inconsistent HMPRs. An integrated procedure to manage the multiplicative consistencies of HMPRs is designed. The proposed methods are also extended to accommodate incomplete HMPRs, and to estimate missing values. Finally, some numerical examples, a comparative analysis with existent approaches, and a simulation analysis are included to illustrate the practicality and effectiveness of the developed models.
\end{abstract}

Index Terms-Consistency ascertaining, hesitant multiplicative preference relations (HMPRs), inconsistency repairing, missing values, weights derivation.

\section{INTRODUCTION}

$\mathbf{I}$ $\mathrm{N}$ DECISION making, the following relations are widely used to represent the preference information of decision makers: multiplicative preference relation (MPR) [1], fuzzy preference relation [2], interval preference relation [3]-[5], intuitionistic preference relation [6]-[9], and linguistic preference relation [10]-[13]. However, these preference relations

Manuscript received March 26, 2020; revised July 19, 2020, November 20, 2020, and April 19, 2021; accepted July 19, 2021. This work was supported in part by the National Natural Science Foundation of China (NSFC) under Grant 71871085, and in part by FEDER Funds provided in the National Spanish Project under Grant PID2019-103880RB-I00. This article was recommended by Associate Editor M. Zhou. (Corresponding authors: Yejun Xu; Enrique Herrera-Viedma.)

Yejun $\mathrm{Xu}$ is with the College of Management and Economics, Tianjin University, Tianjin 300072, China (e-mail: xuyejohn@163.com).

Mengqi Li is with the Business School, Hohai University, Nanjing 211100, China (e-mail: sdbzlm7@163.com).

Francisco Chiclana is with the Andalusian Research Institute in Data Science and Computational Intelligence, University of Granada, 18071 Granada, Spain, and also with the Institute of Artificial Intelligence, School of Computer Science and Informatics, De Montfort University, Leicester LE1 9BH, U.K. (e-mail: chiclana@dmu.ac.uk).

Enrique Herrera-Viedma is with the Andalusian Research Institute in Data Science and Computational Intelligence, University of Granada, 18071 Granada, Spain, and also with the Department of Electrical and Computer Engineering, Faculty of Engineering, King Abdulaziz University, Jeddah 21589, Saudi Arabia (e-mail: viedma@ decsai.ugr.es).

Color versions of one or more figures in this article are available at https://doi.org/10.1109/TSMC.2021.3099862.

Digital Object Identifier 10.1109/TSMC.2021.3099862 do not allow to handle situations where decision makers ascertain the membership of elements with a set of values derived from their hesitancy among several different values. To handle these cases, Torra [14] introduced the concept of the hesitant fuzzy set with elements in the unit interval $[0,1]$. Based on the concept of the hesitant fuzzy set, Xia and Xu [15] used Saaty's analytic hierarchy process (AHP) 1/9-9 scale [1] to further define the concept of hesitant MPR (HMPR), which can vividly simulate both the decision makers' uncertainty and hesitation by allowing preferences to be expressed with hesitant multiplicative elements (HMEs) using the AHP scale.

In recent years, HMPR research has become a hot topic [16]. In particular, it is worth mentioning the HMPR research on priority weights derivation [17]-[20], consistency analysis [21]-[25], and group consensus [21], [26]-[29].

Consistency is one of the key and challenging issues that need to be resolved in decision-making processes. Inconsistent preferences can lead to bad decisions. Thus, methods have been developed to deal with the consistencies of the various preference relations [10], [11], [30]-[39]. Consistency of HMPRs, which can help decision makers to derive reasonable weighting values and decision results, has also received great attention recently with regard to the following two aspects: 1) consistency ascertaining: how to measure the consistency level of an HMPR and 2) inconsistency repairing: how to derive an HMPR with acceptable consistency from an inconsistent HMPR.

So far, research scholars have made some suggestions regarding consistency and the priority derivation of HMPRs. Indeed, Zhang and $\mathrm{Wu}$ [21] defined the multiplicatively consistent HMPR and developed a decision support model for group decision making as per the group consensus level. However, their $\alpha$-normalization and $\beta$-normalization processes reduce or add some additional values to an HME, respectively, which destructs and distorts the decision maker's original judgments. Furthermore, Zhang and Wu [17] introduced the definitions of consistent HMPR and acceptably consistent HMPR, and derived the interval weights from HMPRs based on the $\beta$-normalization process but no inconsistency rectification process was proposed. Meng et al. also defined the consistency of HMPRs in [25], which is based on the assumption of any element in the HMEs forming a consistent MPR. In real applications, it is difficult to provide fully consistent MPRs, which is even more difficult in the case of HMPRs. If the uncertain information provided by a decision maker is 
consistent, then this indicates that such decision maker possesses a strong logic and is sure of his/her information [40]. In other words, he/she knows his/her preferences perfectly well, and therefore he/she is not hesitant about his/her judgments in the HMPR. Mou et al. [23] defined the multiplicative consistency level of HMPRs and developed a method to repair inconsistency, which included a normalized process, and an artificial threshold of acceptable consistency level. Similarly, Nie [24]'s approach is based on a randomly given threshold of the consistency index, which lacks a theoretical basis. Zhang and Guo [41] gave some formulas for calculating the weights of incomplete HMPRs, but they only considered the acceptable consistent incomplete HMPRs and ignored the inconsistent cases that occur in practical problems. Lin et al. [42] constructed a linear programming model to obtain priorities from HMPRs. Additionally, HMPRs have been widely utilized to handle various practical issues, such as the allocation of water conservancy investment of river basins [18], [25], logistics service provider selection [42], and city sustainable development evaluation [43].

The above analysis highlights some research achievements with regard to the consistency of HMPRs. However, there are still some issues that remain to be solved. The research motivations of this article can be summarized as follows.

1) Existing multiplicative consistency research approaches are often hindered with drawbacks related to the changing of the decision makers' original judgments and the optional setting of consistency thresholds. Therefore, it is necessary to answer the following question: what is the multiplicative consistency of HMPRs and how can it be verified?

2) When decision makers hesitate to express their opinions in decision-making problems, a precise priority vector cannot represent the decision makers' hesitation judgments accurately and naturally [17]. Consequently, the following question needs answering: how to generate suitable and realistic weights from an HMPR with multiplicative consistency?

3) Decision makers with allodoxaphobia may hesitate to deal with decision-making problems. Thus, the development of models to help decision makers eliminate their illogical, inconsistent, or unreasonable information could be really useful to decision makers in general, and to allodoxaphobia decision makers in particular. Hence, a question to address is: if an HMPR is inconsistent, how can inconsistency be repaired?

4) There are few papers in the literature reporting on multiplicative consistency measurement, inconsistency level improvement, and weights derivation for incomplete HMPRs, which is addressed in this article.

To answer the above questions, two new multiplicative consistencies of HMPRs, completely multiplicative consistency and weakly multiplicative consistency, respectively, are introduced. Moreover, 0-1 mixed programming models and some algebraic approaches are developed to determine the consistency type for HMPRs. Goal programming methods are proposed to 1) derive priority weights from an HMPR and 2) find the inconsistent elements in an HMPR. This new approach allows decision makers to assign suitable weights to different stages to reflect their preferences in HMPR problems. Subsequently, an efficient and flexible integrated algorithm is designed to test consistency, obtain logical weights, and repair inconsistency of HMPRs, while a novel method to judge the consistency type, estimate missing values, and derive priority vectors from incomplete HMPRs is developed.

The remainder of this article is arranged as follows. Section II introduces the required basic concepts of MPR, hesitant multiplicative sets (HMSs), and HMPR. Two new definitions of consistency of HMPRs are introduced in Section III. Section IV develops methods to ascertain the consistency of HMPRs. In Section V, a priority weight derivation model and an inconsistency repairing method based on multiplicative consistency are proposed. These are used to obtain consistent HMPRs and the reasonable alternatives ranking results. Section VI is devoted to incomplete HMPRs, and two multiplicative consistency-based goal programming models are proposed to assess their unknown values and to ascertain their consistency. Section VII provides three examples, a discussion, and a simulation analysis to show the effectiveness of the developed approaches. Finally, some conclusions are offered in Section VIII.

\section{PRELIMINARIES}

In order to make this article self-contained, some concepts associated with MPRs, HMSs, and HMPRs, which are used throughout this article, are reviewed.

For simplicity, let $X=\left\{x_{1}, \ldots, x_{n}\right\}$ be a finite set of alternatives, and $N=\{1, \ldots, n\}$.

Definition 1 [1]: An MPR $R=\left(r_{i j}\right)_{n \times n} \subset X \times X$ is reciprocal if

$$
r_{i j} \cdot r_{j i}=1, r_{i i}=1, r_{i j} \in[1 / 9,9] \quad \forall i, j \in N .
$$

Definition 2 [1]: An MPR $R=\left(r_{i j}\right)_{n \times n}$ is perfect consistent if

$$
r_{i j}=r_{i k} \cdot r_{k j} \quad \forall i, j, k \in N .
$$

Let $w=\left(w_{1}, \ldots, w_{n}\right)^{T}$ be the weight vector of the set of alternatives $X$, such that $w_{i}>0$, and $\sum_{i=1}^{n} w_{i}=1$. If MPR $R$ on $X$ is perfect consistent, then

$$
r_{i j}=\frac{w_{i}}{w_{j}} \quad \forall i, j \in N .
$$

An MPR is incomplete when some of its elements are missing.

Definition 3 [44]: An MPR $R=\left(r_{i j}\right)_{n \times n}$ is incomplete when some of its elements cannot be given by the decision maker, while the rest of provided preference values, $\Omega$, satisfy the conditions

$$
r_{i j} \cdot r_{j i}=1, r_{i i}=1, r_{i j}>0, \text { for all } r_{i j} \in \Omega \text {. }
$$

Definition 4 [45]: An incomplete MPR $R=\left(r_{i j}\right)_{n \times n}$ is consistent if

$$
r_{i j}=r_{i k} \cdot r_{k j}, \text { for all } r_{i j}, r_{i k}, r_{k j} \in \Omega \text {. }
$$

Motivated by the concepts of the hesitant fuzzy set and MPR, Xia and Xu [15] defined the concept of HMS. 
Definition 5 [15]: An HMS $M$ on $X$ is mathematically expressed as

$$
M=\left\{<x, b_{M}(x)>\mid x \in X\right\}
$$

where $b_{M}(x)$ is a subset of finite cardinality of set $[1 / 9,9]$, which denotes all the possible membership degrees of the element $x \in X$ to the set $M$.

For convenience, $b=b_{M}(x)$ is often called an HME. Motivated by Torra [14], Zhang and Wu [17] defined the upper and lower bounds of an HME.

Definition 6 [17]: The upper and lower bounds of an HME are $h_{i j}^{+}=\max \left\{h_{i j}^{t} \mid t=1, \ldots, l_{h_{i j}}\right\}$ and $h_{i j}^{-}=\min \left\{h_{i j}^{t} \mid t=\right.$ $\left.1, \ldots, l_{h_{i j}}\right\}$, respectively.

Combining HMSs and MPRs, the concept of HMPR is defined.

Definition 7 [15]: An HMPR $H=\left(h_{i j}\right)_{n \times n} \subset X \times X$ is a preference relation with HMEs, $h_{i j}=\left\{h_{i j}^{t} \mid t=1,2, \ldots, l_{h_{i j}}\right\}$, indicating all the possible degrees to which alternative $x_{i}$ is preferred to alternative $x_{j}$ subject to the constraints

$$
h_{i j}^{\sigma(t)} h_{j i}^{\sigma\left(l_{h_{i j}}-t+1\right)}=1, h_{i i}=\{1\}, l_{h_{i j}}=l_{h_{j i}}, i, j \in N
$$

where $h_{i j}^{\sigma(t)}$ denotes the $t$ th smallest element in $h_{i j}$.

Similar to the definition of hesitant fuzzy preference relations discussed by $\mathrm{Xu}$ et al. [46], the values in each HME are ordered from smallest to largest as per Definition 7, which may result in property (7) not to be verified. At the same time, because of the disorder of sets, there is no need to arrange $h_{i j}$ in ascending or descending order. Thus, a revised definition of HMPRs is introduced here.

Definition 8: An HMPR $H=\left(h_{i j}\right)_{n \times n} \subset X \times X$ is a preference relation with HMEs, $h_{i j}=\left\{h_{i j}^{t} \mid t=1,2, \ldots, l_{h_{i j}}\right\}$, indicating the possible degrees to which alternative $x_{i}$ is preferred to alternative $x_{j}$, subject to the following constraints:

$$
h_{i j}^{t} h_{j i}^{l_{h_{i j}}-t+1}=1, h_{i i}=\{1\}, l_{h_{i j}}=l_{h_{j i}}, i, j \in N .
$$

If some elements of an HMPR cannot be given by a decision maker, then an incomplete HMPR results. Zhang and Guo [41] introduced the concept of acceptable incomplete HMPRs.

Definition 9 [41]: An HMPR $H=\left(h_{i j}\right)_{n \times n} \subset X \times X$ is incomplete when some of its HMEs are unknown while its known HMEs $h_{i j}=\left\{h_{i j}^{t} \mid t=1,2, \ldots, l_{h_{i j}}\right\}$ satisfy the constraints

$$
h_{i j}^{t} h_{j i}^{l_{h i j}-t+1}=1, h_{i i}=\{1\}, l_{h_{i j}}=l_{h_{j i}}, \quad i, j \in N .
$$

To improve readability, Table I lists the abbreviations used in this article.

\section{Multiplicative Consistencies of HMPRs}

This section introduces two multiplicative consistency concepts for HMPRs: 1) completely multiplicative consistency and 2) weakly multiplicative consistency.

Definition 10: Let $H=\left(h_{i j}\right)_{n \times n}$ be an HMPR. If there is a complete consistent MPR $R=\left(r_{i j}\right)_{n \times n}$, such that

$$
r_{i j}=r_{i k} \cdot r_{k j}, r_{i j} \in h_{i j} \quad \forall i, j, k \in N
$$

TABLE I

NOMENCLATURE

\begin{tabular}{cc}
\hline Abbreviations & Illustration \\
\hline AHP & Analytic Hierarchy Process \\
MPRs & Multiplicative preference relations \\
HMPRs & Hesitant multiplicative preference relations \\
HMSs & Hesitant multiplicative sets \\
HMEs & Hesitant multiplicative elements \\
CMC & Completely multiplicative consistent \\
WMC & Weakly multiplicative consistent \\
LCR & Length change ratio \\
NAR & Numerical adjustment ratio \\
AD & Absolute deviation \\
LAD & Logarithm absolute deviation \\
DR & Difference ratio \\
\hline
\end{tabular}

then $H$ is called a completely multiplicative consistent (CMC) HMPR and $R$ is a complete consistent MPR in $H$.

The CMC HMPR concept extracts existing elements from the HMPR to form an MPR that satisfies the multiplicative transitivity property (2). As the information provided by a decision maker is uncertain, our goal is "to find the reasonable information in an HMPR." Definition 10 does not rely on Zhang's $\beta$-normalization [26]. Therefore, no elements are added to HMEs. In any case, completely multiplicative consistency is difficult to be verified by an HMPR. Let us consider the following example: when evaluating a set of three alternatives $X=\left\{x_{1}, x_{2}, x_{3}\right\}$, a decision maker expresses that alternative $x_{1}$ is weakly less important than alternative $x_{2}$, and gives the preference value $h_{12}=1 / 2$; while $x_{2}$ is strongly more important than alternative $x_{3}$, and gives the preference value $h_{23}=5$. In the AHP context, if his/her information is consistent, then it should be $h_{13}=h_{12} \times h_{23}=5 / 2$. However, the value $5 / 2$ is not one of the original scale values in the AHP scale set $\{1 / 9, \ldots, 1 / 2,1,2, \ldots, 9\}$. In addition to the above, if the decision maker is unsure about the preference of alternative $x_{1}$ over alternative $x_{3}$ but considers $x_{1}$ more important than $x_{3}$, and gives the following $\operatorname{HME}\{2,3\}$, then it is obviously that his/her preferences are not CMC (5/2 is between 2 and 3). In this case, we could regard the decision maker's information to be close to complete consistent. In our view, because the upper and lower bounds of HMEs produce a range containing all possible decision maker's preference information, the extraction of a consistent MPR from the upper and lower bounds of HMEs is a viable approach. In order to accommodate this scenario, another consistency property of HMPRs is introduced here.

Definition 11: Let $H=\left(h_{i j}\right)_{n \times n}$ be an HMPR. If there is a complete consistent MPR $R=\left(r_{i j}\right)_{n \times n}$ satisfying

$$
r_{i j}=r_{i k} \cdot r_{k j}, h_{i j}^{-} \leq r_{i j} \leq h_{i j}^{+} \quad \forall i, j, k \in N
$$

then $H$ is called a weakly multiplicative consistent (WMC) HMPR and $R$ is a complete consistent MPR in $H$.

Considering the aforementioned relationship between $r_{i j}$ and $w$ as per (3), an equivalent definition of WMC HMPR is given as follows.

Definition 12: An HMPR $H=\left(h_{i j}\right)_{n \times n}$ is called a WMC HMPR, if there exists a weight vector $w=\left(w_{1}, \ldots, w_{n}\right)^{T}$, 


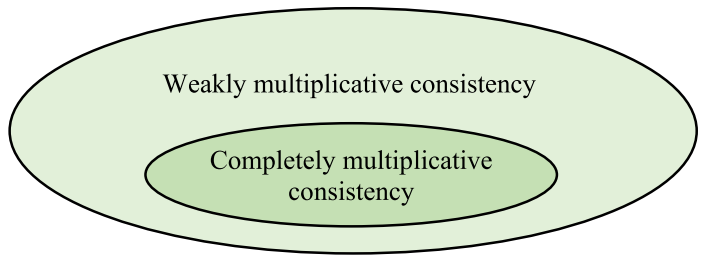

Fig. 1. Relationship of the two multiplicative consistencies for HMPRs.

such that

$$
h_{i j}^{-} \leq \frac{w_{i}}{w_{j}} \leq h_{i j}^{+} \quad \forall i, j \in N .
$$

The reciprocity property of HMPRs allows the above definition to be rewritten equivalently as follows.

Definition 13: An HMPR $H=\left(h_{i j}\right)_{n \times n}$ is called a WMC HMPR if there is a weighting vector $w=\left(w_{1}, \ldots, w_{n}\right)^{T}$, such that

$$
h_{i j}^{-} \leq \frac{w_{i}}{w_{j}} \leq h_{i j}^{+}, i=1,2, \ldots, n, j=i+1, \ldots, n .
$$

It is obvious that a CMC HMPR is a special case of WMC HMPR, while a WMC HMPR might not necessarily be a CMC HMPR (see Fig. 1).

\section{Consistency Ascertaining}

An important question to answer is whether an HMPR is CMC or WMC.

The direct verification of the CMC property as per Definition 11 is not an easy task. In order to facilitate calculation, the following 0-1 indicator variables of HMEs $h_{i j}$ are introduced: $\alpha_{i j}^{t}=\left\{\begin{array}{ll}1, & \text { if } h_{i j}^{t} \in h_{i j} \text { is chosen } \\ 0, & \text { otherwise }\end{array}\right.$. Each element in $h_{i j}$ can be expressed as follows: $\prod_{t=1}^{h_{i j}}\left(h_{i j}^{t}\right)^{\alpha_{i j}^{t}}$ with $\sum_{t=1}^{l_{h_{i j}}} \alpha_{i j}^{t}=1$.

According to Definition 10, if $H$ is a CMC HMPR, then

$$
\prod_{t=1}^{l_{h_{i j}}}\left(h_{i j}^{t}\right)^{\alpha_{i j}^{t}}=\prod_{t=1}^{l_{h_{i k}}}\left(h_{i k}^{t}\right)^{\alpha_{i k}^{t}} \times \prod_{t=1}^{l_{h_{k j}}}\left(h_{k j}^{t}\right)^{\alpha_{k j}^{t}} .
$$

This is equivalent to

$$
\sum_{t=1}^{l_{h_{i j}}} \alpha_{i j}^{t} \log \left(h_{i j}^{t}\right)-\sum_{t=1}^{l_{h_{i k}}} \alpha_{i k}^{t} \log \left(h_{i k}^{t}\right)-\sum_{t=1}^{l_{h k j}} \alpha_{k j}^{t} \log \left(h_{k j}^{t}\right)=0 .
$$

As aforementioned, (15) does not always hold. We relax (15) appropriately with the introduction of non-negative deviation numbers $d_{i j k}^{-}$and $d_{i j k}^{+} \forall i, j, k \in N$

$$
\begin{aligned}
& \sum_{t=1}^{l_{h_{i j}}} \alpha_{i j}^{t} \log \left(h_{i j}^{t}\right)-\sum_{t=1}^{l_{h_{i k}}} \alpha_{i k}^{t} \log \left(h_{i k}^{t}\right)-\sum_{t=1}^{l_{h_{k j}}} \alpha_{k j}^{t} \log \left(h_{k j}^{t}\right) \\
& \quad-d_{i j k}^{+}+d_{i j k}^{-}=0 .
\end{aligned}
$$

Equation (16) becomes (15) iff $d_{i j k}^{-}=d_{i j k}^{+}=0$. Thus, the following 0-1 mixed programming model is established to ascertain the completely multiplicative consistency property of HMPRs

$$
\begin{aligned}
& \text { (M-1) } J_{1}=\min \sum_{k=1}^{n} \sum_{i=1, i \neq k j=1, i \neq j, j \neq k}^{n} \sum_{i j k}^{n}\left(d_{i j k}^{-}\right)
\end{aligned}
$$

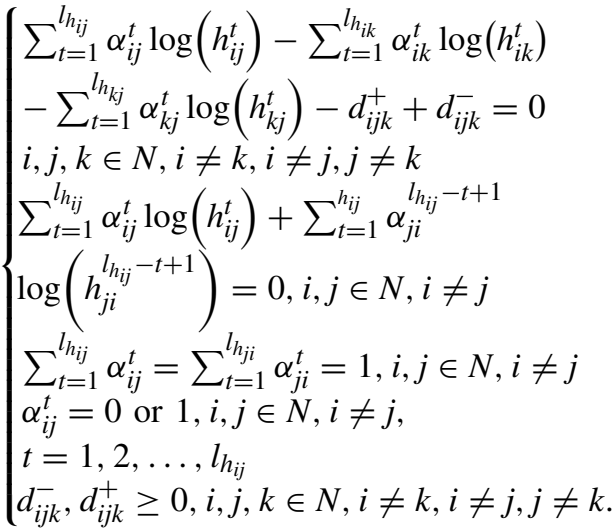

By solving (M-1), if $J_{1}=0$ for all $i, j$ with $i \neq j$ and each $t=1,2 \ldots, l_{h_{i j}}$, then $H$ is CMC; otherwise, $H$ is not CMC.

The reciprocity of $H$ means that (M-1) can be equivalently rewritten as

$$
\begin{aligned}
& \text { (M-2) } J_{2}=\min \sum_{i=1}^{n-1} \sum_{k=i+1}^{j-1} \sum_{j=k+1}^{n}\left(d_{i j k}^{-}+d_{i j k}^{+}\right) \\
& \text {s.t. }\left\{\begin{array}{l}
\sum_{t=1}^{l_{h i j}} \alpha_{i j}^{t} \log \left(h_{i j}^{t}\right)-\sum_{t=1}^{l_{h i k}} \alpha_{i k}^{t} \log \left(h_{i k}^{t}\right) \\
-\sum_{t=1}^{l_{h k j}} \alpha_{k j}^{t} \log \left(h_{k j}^{t}\right)-d_{i j k}^{+}+d_{i j k}^{-}=0 \\
i, j, k \in N, i<k<j \\
\sum_{t=1}^{l_{h i j}} \alpha_{i j}^{t} \log \left(h_{i j}^{t}\right)+\sum_{t=1}^{h_{i j}} \alpha_{j i}^{l_{h i}-t+1} \\
\log \left(h_{j i}^{l_{h i j}-t+1}\right)=0, i, j \in N, i \neq j \\
\sum_{h_{i j}}^{l_{i j}} \alpha_{i j}^{t}=\sum_{t=1}^{l_{h j i}} \alpha_{j i}^{t}=1, i, j \in N, i<j \\
\alpha_{i j}^{t}=0 \text { or } 1, i, j \in N, i<j, \\
t=1,2, \ldots, l_{h_{i j}} \\
d_{i j k}^{-}, d_{i j k}^{+} \geq 0, i, j, k \in N, i<k<j .
\end{array}\right.
\end{aligned}
$$

The following result proves the validity of model (M-2) to ascertain the completely multiplicative consistency property of HMPRs.

Theorem 1: An HMPR $H$ is a CMC HMPR iff $J_{2}=0$.

Proof (Sufficiency): If $J_{2}=0$, then $d_{i j k}^{-}=d_{i j k}^{+}=0 \forall i, j, k \in$ $N$ and (16) reduces to (15). Thus, $H$ is CMC.

Necessary: If $H$ is CMC, (15) holds and $d_{i j k}^{-}=d_{i j k}^{+}=0$ in (16), which implies that $J_{2}=0$.

When $H$ is a CMC HMPR, a complete consistent MPR can be derived by solving (M-2). On the contrary, if $H$ is not a CMC HMPR, in the following, some algebraic methods are proposed to detect whether it is a WMC HMPR.

Theorem 2: An HMPR $H=\left(h_{i j}\right)_{n \times n}$ is a WMC HMPR iff

$$
\max _{k}\left\{h_{i j}^{-}, h_{i k}^{-} h_{k j}^{-}\right\} \leq \min _{k}\left\{h_{i j}^{+}, h_{i k}^{+} h_{k j}^{+}\right\} \quad \forall i, j, k \in N .
$$

Proof: If $H$ is a WMC HMPR, then there is a complete consistent MPR $R=\left(r_{i j}\right)_{n \times n}$ such that

$$
h_{i j}^{-} \leq r_{i j} \leq h_{i j}^{+} \quad \forall i, j \in N
$$




$$
\begin{aligned}
& h_{i k}^{-} \leq r_{i k} \leq h_{i k}^{+} \quad \forall i, k \in N \\
& h_{k j}^{-} \leq r_{k j} \leq h_{k j}^{+} \quad \forall k, j \in N .
\end{aligned}
$$

Multiplying (19) by (20), we have

$$
h_{i k}^{-} h_{k j}^{-} \leq r_{i j} \leq h_{i k}^{+} h_{k j}^{+} \quad \forall i, j, k \in N
$$

Since (21) holds for any $k \in N$, it is $\max _{k}\left\{h_{i j}^{-}, h_{i k}^{-} h_{k j}^{-}\right\} \leq$ $\min _{k}\left\{h_{i j}^{+}, h_{i k}^{+} h_{k j}^{+}\right\}$for all $i, j, k \in N$.

Conversely, if (17) holds for all $i, j, k \in N$, there exists a complete consistent MPR $R=\left(r_{i j}\right)_{n \times n}$ satisfying $r_{i j}=r_{i k} \cdot r_{k j}$, $h_{i j}^{-} \leq r_{i j} \leq h_{i j}^{+} \forall i, j, k \in N$. By Definition $11, H$ is a WMC HMPR.

As per (17), we have the following equivalence theorem to ascertain the weakly multiplicative consistency property of HMPRs.

Theorem 3: An HMPR $H=\left(h_{i j}\right)_{n \times n}$ is a WMC HMPR iff

$$
\bigcap_{k=1}^{n}\left[h_{i k}^{-} h_{k j}^{-}, h_{i k}^{+} h_{k j}^{+}\right] \neq \varnothing \quad \forall i, j, k \in N .
$$

Proof: We only need to prove that (17) and (22) are equivalent. Suppose that $H$ is a WMC HMPR, then there is a complete consistent MPR $R=\left(r_{i j}\right)_{n \times n}$ such that

$$
\begin{aligned}
& h_{i j}^{-} \leq r_{i j} \leq h_{i j}^{+} \quad \forall i, j \in N \\
& h_{i k}^{-} \leq r_{i k} \leq h_{i k}^{+} \quad \forall i, k \in N \\
& h_{k j}^{-} \leq r_{k j} \leq h_{k j}^{+} \quad \forall k, j \in N .
\end{aligned}
$$

Therefore, it is

$$
h_{i k}^{-} h_{k j}^{-} \leq r_{i j} \leq h_{i k}^{+} h_{k j}^{+} \quad \forall i, j, k \in N .
$$

Since (26) holds for any $k \in N$, it is $r_{i j} \in \bigcap_{k=1}^{n}\left[h_{i k}^{-} h_{k j}^{-}, h_{i k}^{+} h_{k j}^{+}\right] \neq \varnothing$, which is equivalent to $\max _{k}\left\{h_{i j}^{-}, h_{i k}^{-} h_{k j}^{-}\right\} \leq \min _{k}\left\{h_{i j}^{+}, h_{i k}^{+} h_{k j}^{+}\right\}$. By Theorem $2, H$ is a WMC HMPR, which completes the proof of Theorem 3.

The below interval MPR definition is needed for Theorem 4, which is an equivalent result to Theorems 2 and 3, to ascertain the weakly multiplicative consistency property of HMPRs. Recall that given two interval numbers $\bar{x}=\left[x^{-}, x^{+}\right]$and $\bar{y}=$ $\left[y^{-}, y^{+}\right]$with $x^{-}, y^{-}>0$, their product is $\bar{x} \cdot \bar{y}=\left[x^{-} y^{-}, x^{+} y^{+}\right]$.

Definition 14 [47]: An interval MPR $\bar{H}=\left(\bar{h}_{i j}\right)_{n \times n}$ is a preference relation with elements $\bar{h}_{i j}=\left[h_{i j}^{-}, h_{i j}^{+}\right]$verifying: $0<h_{i j}^{-} \leq h_{i j}^{+}, h_{i j}^{-} h_{j i}^{+}=1, h_{i j}^{+} h_{j i}^{-}=1$. The element $\bar{h}_{i j}$ is called the interval preference ratio and denotes that alternative $x_{i}$ is between $h_{i j}^{-}$and $h_{i j}^{+}$times as important as alternative $x_{j}$.

Notice that given an HMPR $H=\left(h_{i j}\right)_{n \times n}$, the interval MPR $\bar{H}=\left(\bar{h}_{i j}\right)_{n \times n}$ with elements $\bar{h}_{i j}=\left[h_{i j}^{-}, h_{i j}^{+}\right]$can be constructed.

Theorem 4: An HMPR $H=\left(h_{i j}\right)_{n \times n}$ is a WMC HMPR iff $\bar{H}=\left(\bar{h}_{i j}\right)_{n \times n}, \bar{h}_{i j}=\left[h_{i j}^{-}, h_{i j}^{+}\right]$, satisfies

$$
\bigcap_{k=1}^{n}\left(\bar{h}_{i k} \bar{h}_{k j}\right) \neq \varnothing \quad \forall i, j, k \in N .
$$

Proof (Sufficiency): If $\bigcap_{k=1}^{n}\left(\bar{h}_{i k} \bar{h}_{k j}\right) \neq \varnothing$ for all $i, j, k \in$ $N$, then it is $\bigcap_{k=1}^{n}\left(\bar{h}_{i k} \bar{h}_{k j}\right)=\left[p_{i j}^{-}, p_{i j}^{+}\right]$. Thus, it is $\max _{k}\left\{h_{i j}^{-}, h_{i k}^{-} h_{k j}^{-}\right\} \leq p_{i j}^{-} \leq p_{i j}^{+} \leq \min _{k}\left\{h_{i j}^{+}, h_{i k}^{+} h_{k j}^{+}\right\}$, i.e., Theorem 2 is true and $H$ is WMC.
Necessary: If $H$ is a WMC HMPR, then there is a complete consistent MPR $R=\left(r_{i j}\right)_{n \times n}$ satisfying $h_{i j}^{-} \leq r_{i j} \leq$ $h_{i j}^{+}$and $r_{i j}=r_{i k} \cdot r_{k j} \in \bar{h}_{i k} \bar{h}_{k j} \forall i, j, k \in N$. Therefore, $\bigcap_{k=1}^{n}\left(\bar{h}_{i k} \bar{h}_{k j}\right) \neq \varnothing$.

The reciprocity of HMPRs means that when ascertaining the validity of the above results only the elements of the upper or lower part of an HMPR are to be considered.

\section{Goal Programming ApProach to Priority WEIGHT DERIVATION AND INCONSISTENCY REPAIRING OF HMPR}

Consistency is a key property of preference relations, so it is natural to generate priority weights of alternatives from consistent HMPRs. In this section, the following two research questions will be answered: 1) How to generate a priority weight vector from a consistent HMPR? and 2) How to rectify the inconsistency of an HMPR?

To answer these questions, effective optimization models based on multiplicative consistency are established: 1) to test the weakly multiplicative consistency property; 2) to derive priority weights of alternatives; and 3) to repair the inconsistency of a given HMPR.

To find out whether a given HMPR $H=\left(h_{i j}\right)_{n \times n}$ is WMC, non-negative deviation values $d_{i j}^{-}$and $d_{i j}^{+}$are introduced in (13)

$$
\begin{gathered}
h_{i j}^{-}-d_{i j}^{-} \leq \frac{w_{i}}{w_{j}} \leq h_{i j}^{+}+d_{i j}^{+}, i=1,2, \ldots, n \\
j=i+1, \ldots, n .
\end{gathered}
$$

Clearly, $H$ is WMC iff $d_{i j}^{-}$and $d_{i j}^{+}$are 0 in (28), for $i=1, \ldots, n, j=i+1, \ldots, n$. Therefore, the sum of these deviations is used as the objective function of the following optimization model:

$$
\begin{aligned}
& \text { (M-3) } J_{3}=\min \sum_{i=1}^{n-1} \sum_{j=i+1}^{n}\left(d_{i j}^{-}+d_{i j}^{+}\right) \\
& \text {s.t. }\left\{\begin{array}{l}
\frac{w_{i}}{w_{j}}+d_{i j}^{-} \geq h_{i j}^{-}, i=1,2, \ldots, n-1 \\
j=i+1, \ldots, n \\
\frac{w_{i}}{w_{j}}-d_{i j}^{+} \leq h_{i j}^{+}, i=1,2, \ldots, n-1, \\
j=i+1, \ldots, n \\
\sum_{i=1}^{n} w_{i}=1 \\
w_{i} \geq 0, i=1,2, \ldots, n \\
d_{i j}^{-}, d_{i j}^{+} \geq 0, i=1,2, \ldots, n-1, \\
j=i+1, \ldots, n .
\end{array}\right.
\end{aligned}
$$

The following result proves the validity of model (M-3) to ascertain the weakly multiplicative consistency property of HMPRs.

Theorem 5: An HMPR $H=\left(h_{i j}\right)_{n \times n}$ is a WMC HMPR iff $J_{3}=0$.

Proof (Necessary): If $H$ is a WMC HMPR, then (13) holds and it is $d_{i j}^{-}=d_{i j}^{+}=0$ in (28), which implies that $J_{3}=0$.

Sufficiency: If $J_{3}=0$, then $d_{i j}^{-}=d_{i j}^{+}=0 \forall i, j \in N$, and (28) becomes (13). Hence, $H$ is a WMC HMPR.

Model (M-3) provides an alternative way, but equivalent to (13), to ascertain the weakly multiplicative consistency property of HMPRs. Unlike the algebraic operations in 
Section IV, model (M-3) generates the priority weights of alternatives directly from the HMPR. In any case, when $J_{3}=0, H$ is WMC but not necessarily CMC, which can be ascertained with model (M-2).

Since a nonlinear programming model may have multiple solutions, there may be more than one set of weights $w_{i}$ with $J_{3}=0$ in (M-3). As a result, (M-3) main aims are to ascertain whether an HMPR has the weakly multiplicative consistency property and to repair inconsistency, but not to derive the priority weight vector. Hence, it is only necessary to observe the value of $J_{3}$ to test if the consistency type is WMC. Thus, a more reasonable and reliable method to derive the weights of alternatives is needed.

When an HMPR is consistent, model (M-3) results in priority weights of alternatives as single values in the unit interval. However, following the argument provided in [17], interval priority weights are more natural and reasonable than precise weights for hesitant judgments provided by decision makers. Therefore, to generate interval priority weights of alternatives from consistent HMPRs, the below lower and upper approximation models are proposed

$$
\begin{aligned}
& \text { (M-4) } w_{i}^{-}=\min w_{i} \\
& \text { s.t. }\left\{\begin{array}{l}
\frac{w_{i}}{w_{j}} \geq h_{i j}^{-}, i=1,2, \ldots, n-1 \\
j=i+1, \ldots, n \\
\frac{w_{i}}{w_{j}} \leq h_{i j}^{+}, i=1,2, \ldots, n-1 \\
j=i+1, \ldots, n \\
\sum_{i=1}^{n} w_{i}=1 \\
w_{i} \geq 0, i=1,2, \ldots, n .
\end{array}\right. \\
& \text { (M-5) } w_{i}^{+}=\max w_{i} \\
& \text { s.t. }\left\{\begin{array}{l}
\frac{w_{i}}{w_{j}} \geq h_{i j}^{-}, i=1,2, \ldots, n-1 \\
j=i+1, \ldots, n \\
\frac{w_{i}}{w_{j}} \leq h_{i j}^{+}, i=1,2, \ldots, n-1 \\
j=i+1, \ldots, n \\
\sum_{i=1}^{n} w_{i}=1 \\
w_{i} \geq 0, i=1,2, \ldots, n .
\end{array}\right.
\end{aligned}
$$

Given a WMC HMPR, solving models (M-4) and (M-5) will result in unique optimal interval priority weights of alternatives $w_{i}=\left[w_{i}^{-}, w_{i}^{+}\right] \forall i \in N$. Thus, if an HMPR is not consistent, it is necessary first to repair its inconsistency. In the following, an inconsistency repairing method is proposed. The principles of the modification are two: 1) to reduce the total adjustments of an HMPR, and 2) not to increase the number of the values in the adjusted HMPR with respect to the original HMPR.

Given an inconsistency HMPR, model (M-3) allows to identify the inconsistent elements. Therefore, it can guide the inconsistency repairing process as described as follows.

1) If $J_{3} \neq 0$, then there are optimal nonzero deviations $d_{i j}^{-}$and $d_{i j}^{+}$when solving (M-3), which corresponds to HMPR inconsistent elements. Indeed, if $d_{i_{0} j_{0}}^{+(-)} \neq 0$, then $h_{i_{0} j_{0}}$ is an inconsistent element. With regard to the inconsistent element, its range changes from $\left[h_{i j}^{-}, h_{i j}^{+}\right]$to $\left[h_{i j}^{-}-d_{i j}^{-}, h_{i j}^{+}+d_{i j}^{+}\right]$. In other words, the upper and lower bounds of HME are replaced by the new values $h_{i j}^{+}+d_{i j}^{+}$ and $h_{i j}^{-}-d_{i j}^{-}$, while the other values remain unchanged,

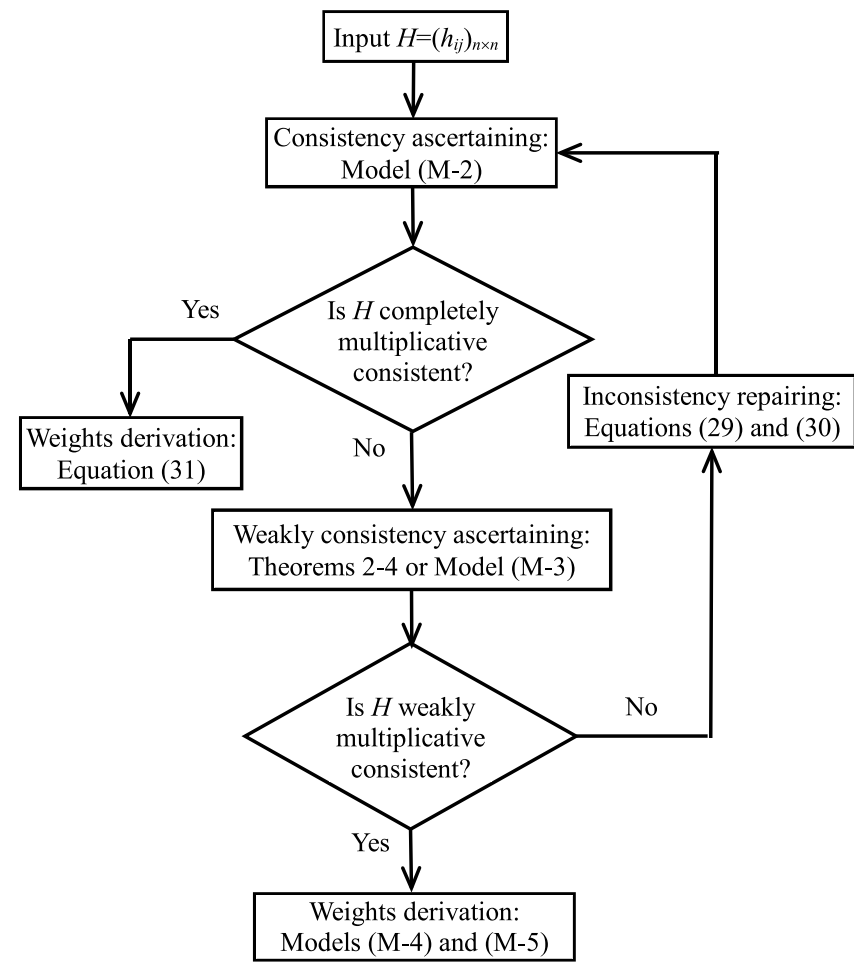

Fig. 2. Process of consistency ascertaining, inconsistency repairing, and weights derivation for HMPRs.

that is

$$
\bar{h}_{i j}=\left\{\begin{array}{l}
\left\{h_{i j}^{-}-d_{i j}^{-} \text {or } h_{i j}^{+}+d_{i j}^{+}\right\} \\
l_{h_{i j}=1} h_{i j}^{-}-d_{i j}^{-}, h_{i j}^{\sigma(2)}, \ldots, h_{i j}^{\sigma\left(l_{\left.h_{i j}-1\right)}, h_{i j}^{+}+d_{i j}^{+}\right\}} \\
l_{h_{i j}} \neq 1 .
\end{array}\right.
$$

Thus, a new modified HMPR $\overline{\mathrm{H}}$ is obtained

$$
\left(\bar{h}_{i j}, \bar{h}_{j i}\right)= \begin{cases}\left(\bar{h}_{i j}, 1 / \bar{h}_{i j}\right), & \text { if } h_{i j} \text { is the inconsistent } \\ \left(h_{i j}, h_{j i}\right), & \text { element } \\ \text { otherwise. }\end{cases}
$$

2) In (29), there are two cases for adjusting the inconsistent elements. Notice that when there is only one element in the HME, the original value is replaced by the modified value. When there are two or more elements in the HME, the lower and upper bound values are replaced and the rest of values are unchanged. This approach maintains the original number of values in each HME, and preserves most of the decision maker's original preferences because only the inconsistent elements are adjusted.

3) After improving the consistency of the HMPR, the priority weight vector derived from the newly adjusted HMPR satisfies (13), and it is $J_{3}=0$. Consequently, (29) and (30) convert an inconsistent HMPR into a consistent HMPR. 
Algorithm 1: Algorithms for consistency ascertaining, inconsistency repairing and weights derivation of HMPRs.

Step 1. Given an HMPR $H$, check completely multiplicative consistency property with model (M-2). If $H$ is CMC, go to Step 3A; otherwise, go to next step.

Step 2. Check weakly multiplicative consistency property by (17), (22), (27) or model (M-3). If $H$ is WMC, go to Step 3B; otherwise, go to Step 4.

Step 3. Priority weights derivation and ranking of alternatives.

Step 3A. (Following Step 1).

Derive the priority weights with the Logarithmic Least Squares Method [48, 49]:

$$
w_{i}=\left(\prod_{j=1}^{n} r_{i j}\right)^{1 / n} / \sum_{i=1}^{n}\left(\prod_{j=1}^{n} r_{i j}\right)^{1 / n}, i=1,2, \ldots, n
$$

and go to Step 5 .

Step 3B. (Following Step 2).

Generate the interval weights with models (M-4) and (M-5). Alternatives are ranked according to their priority weights ranking, via the degree of possibility of $w_{i} \geq w_{j}[5,50]$ :

$$
\begin{aligned}
p\left(w_{i} \geq w_{j}\right) & =\frac{\max \left\{0, w_{i}^{+}-w_{j}^{-}\right\}-\max \left\{0, w_{i}^{-}-w_{j}^{+}\right\}}{w_{i}^{+}-w_{i}^{-}+w_{j}^{+}-w_{j}^{-}} \\
p_{i} & =\sum_{j=1}^{n} p_{i j}, i \in N
\end{aligned}
$$

and $p_{i j}=p\left(w_{i} \geq w_{j}\right)$.

Interval weights are ranked using $p_{i}$ values, i.e. $w_{i} \stackrel{p\left(w_{i} \geq w_{j}\right)}{\succ} w_{j}$ iff $p_{i}>p_{j}$. Go to Step 5 .

Step 4. Solve model (M-3), and repair inconsistency with (29); construct the newly adjusted HMPR with (30). Go to Step 1.

Step 5. End.

In what follows, an integrated algorithm to ascertain consistency, inconsistency repairing, and priority weights derivation for HMPRs is proposed, with corresponding flowchart depicted in Fig. 2.

\section{INCOMPLETE HMPRS}

In a decision-making problem, decision makers may omit some judgments, i.e., some information may be unknown. Hence, a key problem to address is the estimation of missing information. With respect to incomplete HMPRs, this section extends two multiplicative consistency concepts of complete HMPRs to the case of incomplete HMPRs and utilizes two multiplicative consistency-based goal programming models: 1) to estimate their missing HME preference values, and 2) to ascertain the type of multiplicative consistency property that is verified.

Let $H=\left(h_{i j}\right)_{n \times n}$ be an incomplete HMPR. The notation $h_{i j}=x$ is used to represent that $h_{i j}$ is not given by the decision maker. To incorporate (16) into incomplete HMPRs, the following indicator functions for an incomplete HMPR $H$ are introduced:

$$
\begin{aligned}
\delta_{i j} & = \begin{cases}1, & h_{i j} \neq x \\
0, & h_{i j}=x\end{cases} \\
\delta_{i j k} & = \begin{cases}1, & \delta_{i j} \delta_{i k} \delta_{k j}=1 \\
0, & \text { otherwise. }\end{cases}
\end{aligned}
$$

When $h_{i j}, h_{i k}$, and $h_{k j}$ are all known it is $\delta_{i j k}=1$. Then, (16) for an incomplete HMPR can be rewritten as

$$
\begin{aligned}
& \delta_{i j k}\left(\sum_{t=1}^{l_{h_{i j}}} \alpha_{i j}^{t} \log \left(h_{i j}^{t}\right)-\sum_{t=1}^{l_{h_{i k}}} \alpha_{i k}^{t} \log \left(h_{i k}^{t}\right)-\sum_{t=1}^{l_{h_{k j}}} \alpha_{k j}^{t} \log \left(h_{k j}^{t}\right)\right) \\
& -\varepsilon_{i j k}^{+}+\varepsilon_{i j k}^{-}=0 .
\end{aligned}
$$

Consequently, to ascertain the completely multiplicative consistency property of incomplete HMPRs, the following 0-1 mixed programming model is constructed:

$$
\begin{aligned}
& \text { (M-6) } J_{6}=\min \sum_{k=1}^{n} \sum_{i=1, i \neq k j=1,}^{n} \sum_{i \neq j, j \neq k}^{n}\left(\varepsilon_{i j k}^{-}+\varepsilon_{i j k}^{+}\right)
\end{aligned}
$$

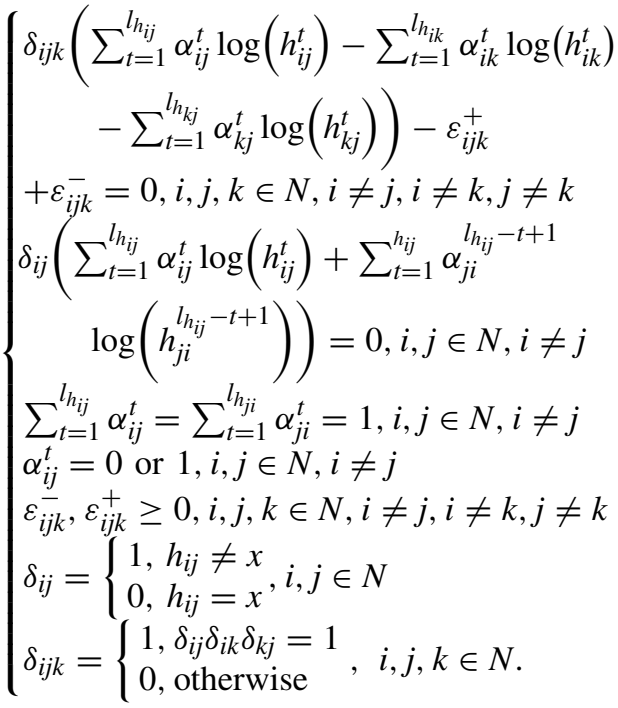

This model can be equivalently simplified as follows:

$$
\begin{aligned}
& \text { (M-7) } J_{7}=\min \sum_{k=1}^{n} \sum_{i=1}^{n-1} \sum_{j=i+1}^{n}\left(\varepsilon_{i j k}^{-}+\varepsilon_{i j k}^{+}\right)
\end{aligned}
$$

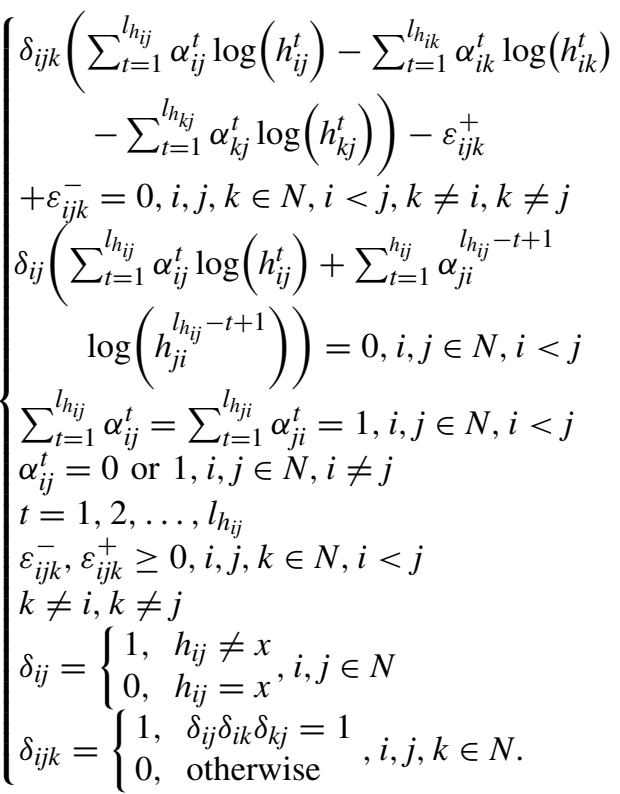

The following result proves the validity of model (M-7) to ascertain the completely multiplicative consistency property of incomplete HMPRs. 
Theorem 6: An incomplete HMPR $H$ is CMC iff $J_{7}=0$.

Proof (Necessary): If an incomplete HMPR $H$ is CMC, then (15) holds for all known elements, that is, $\varepsilon_{i j k}^{-}=\varepsilon_{i j k}^{+}=0$ in (34). Therefore, $J_{7}=0$.

Sufficiency: If an incomplete HMPR $H$ has $J_{7}=0$, then $\varepsilon_{i j k}^{-}=\varepsilon_{i j k}^{+}=0$ for all known elements, and (34) becomes (15). Thus, the incomplete HMPR $H$ is CMC.

The following example illustrates the process of estimating missing values and determining the consistency of incomplete HMPRs with model (M-7).

Example 1: Consider the incomplete HMPR $H_{1}$ (adapted from [41])

$$
H_{1}=\left(\begin{array}{cccc}
\{1\} & \left\{\frac{1}{2}, 1\right\} & \left\{\frac{1}{3}, \frac{1}{2}\right\} & \{1\} \\
\{1,2\} & \{1\} & \{1,2,3\} & \{2\} \\
\{2,3\} & \left\{\frac{1}{3}, \frac{1}{2}, 1\right\} & \{1\} & x \\
\{1\} & \left\{\frac{1}{2}\right\} & x & \{1\} .
\end{array}\right) .
$$

Solving model (M-7) gives $J_{7}=0$. Thus, the incomplete HMPR $H_{1}$ is CMC. At the same time, the missing HMEs obtained are $h 34=\{2\}$ and $h_{43}=\{1 / 2\}$, and there exists a complete consistent MPR $R_{1}$

$$
R_{1}=\left(\begin{array}{cccc}
1 & \frac{1}{2} & \frac{1}{2} & 1 \\
2 & 1 & 1 & 2 \\
2 & 1 & 1 & 2 \\
1 & \frac{1}{2} & \frac{1}{2} & 1
\end{array}\right)
$$

Thus, $H_{1}$ is transformed into the below complete HMPR

$$
H_{1}^{\prime}=\left(\begin{array}{cccc}
\{1\} & \left\{\frac{1}{2}, 1\right\} & \left\{\frac{1}{3}, \frac{1}{2}\right\} & \{1\} \\
\{1,2\} & \{1\} & \{1,2,3\} & \{2\} \\
\{2,3\} & \left\{\frac{1}{3}, \frac{1}{2}, 1\right\} & \{1\} & \{2\} \\
\{1\} & \left\{\frac{1}{2}\right\} & \left\{\frac{1}{2}\right\} & \{1\}
\end{array}\right) .
$$

The proposed model is more reasonable and effective than Sahu and Gupta's model [22], since their $\beta$-normalization method is superfluous, and no additional elements are added to HMEs. In addition, the proposed model can determine the consistency type of incomplete HMPRs, while Sahu and Gupta's model fails to do so.

When the incomplete HMPR $H$ is not CMC, its weakly multiplicative consistency property is considered. Similar to Theorems 2-4 of Section IV, the following results for incomplete HMPRs are provided.

Theorem 7: An incomplete HMPR $H=\left(h_{i j}\right)_{n \times n}$ is WMC iff for all known elements

$$
\max _{k}\left\{h_{i j}^{-}, h_{i k}^{-} h_{k j}^{-}\right\} \leq \min _{k}\left\{h_{i j}^{+}, h_{i k}^{+} h_{k j}^{+}\right\}
$$

or, equivalently

$$
\bigcap_{k=1}^{n}\left[h_{i k}^{-} h_{k j}^{-}, h_{i k}^{+} h_{k j}^{+}\right] \neq \varnothing
$$

is verified.

Given an incomplete HMPR, $H=\left(h_{i j}\right)_{n \times n}$, its associated incomplete interval MPR $\bar{H}=\left(\bar{h}_{i j}\right)_{n \times n}$ has elements: $\bar{h}_{i j}=$ $\left[h_{i j}^{-}, h_{i j}^{+}\right]$if $\bar{h}_{i j}$ is known; otherwise, $\bar{h}_{i j}=x$ is unknown. Let $\Omega$ be the set of all the known elements in $\bar{H}$.
Theorem 8: An incomplete HMPR $H=\left(h_{i j}\right)_{n \times n}$ is WMC iff

$$
\bigcap_{k=1}^{n}\left(\bar{h}_{i k} \bar{h}_{k j}\right) \neq \varnothing, \text { for all } \bar{h}_{i j} \in \Omega \text {. }
$$

The following optimization model for incomplete HMPRs can be established, based on the weakly multiplicative consistency property, to estimate the missing information and to test consistency:

$$
\begin{aligned}
& \text { (M-8) } J_{8}=\min \sum_{i=1}^{n-1} \sum_{j=i+1}^{n}\left(\varepsilon_{i j}^{-}+\varepsilon_{i j}^{+}\right) \\
& \text {s.t. }\left\{\begin{array}{l}
\delta_{i j}\left(\frac{w_{i}}{w_{j}}+\varepsilon_{i j}^{-}-h_{i j}^{-}\right) \geq 0 \\
i=1,2, \ldots, n-1, j=i+1, \ldots, n \\
\delta_{i j}\left(\frac{w_{i}}{w_{j}}-\varepsilon_{i j}^{+}-h_{i j}^{+}\right) \leq 0 \\
i=1,2, \ldots, n-1, j=i+1, \ldots, n \\
\sum_{i=1}^{n} w_{i}=1 \\
w_{i} \geq 0, i \in N \\
\delta_{i j}=\left\{\begin{array}{l}
1, h_{i j} \neq x \\
0, h_{i j}=x
\end{array}, i, j \in N\right. \\
\varepsilon_{i j}^{-}, \varepsilon_{i j}^{+} \geq 0, i=1,2, \ldots, n-1 \\
j=i+1, \ldots, n .
\end{array}\right.
\end{aligned}
$$

Solving model (M-8), the incomplete HMPR is WMC when $J_{8}=0$, in which case, via (3), its missing elements can be estimated. As before, an example is provided below to illustrate the weakly multiplicative consistency-based HMPR completion process.

Example 2: Consider the incomplete HMPR $\mathrm{H}_{2}$

$$
H_{2}=\left(\begin{array}{cccc}
\{1\} & \left\{\frac{3}{7}\right\} & \left\{\frac{3}{7}, \frac{3}{2}\right\} & \{1\} \\
\left\{\frac{7}{3}\right\} & \{1\} & \left\{\frac{3}{7}, \frac{3}{2}\right\} & x \\
\left\{\frac{2}{3}, \frac{7}{3}\right\} & \left\{\frac{2}{3}, \frac{7}{3}\right\} & \{1\} & \left\{\frac{2}{3}, \frac{7}{3}\right\} \\
\{1\} & x & \left\{\frac{3}{7}, \frac{3}{2}\right\} & \{1\}
\end{array}\right) .
$$

Solving model $(\mathrm{M}-7)$ gives $J_{7}=2.4692 \neq 0$. Thus, $H_{2}$ is not CMC, and Theorem 7 is used to check whether $\mathrm{H}_{2}$ is WMC. For all known elements, (36) yields

$$
\begin{array}{r}
\bigcap_{k=1}^{4}\left[h_{1 k}^{-} h_{k 3}^{-}, h_{1 k}^{+} h_{k 3}^{+}\right]=\left[\frac{3}{7}, \frac{3}{2}\right] \bigcap\left[\frac{9}{49}, \frac{9}{14}\right] \bigcap\left[\frac{3}{7}, \frac{3}{2}\right] \\
\bigcap\left[\frac{3}{7}, \frac{3}{2}\right]=\left[\frac{9}{49}, \frac{9}{14}\right] \neq \varnothing .
\end{array}
$$

Thus, the incomplete HMPR $\mathrm{H}_{2}$ is WMC. Notice that this can also be verified using (35) as shown in Table II.

Solving model (M-8) gives $J_{8}=0$, and the missing HMEs are estimated as $h_{24}=\{7 / 3\}$ and $h_{42}=\{3 / 7\}$. The following complete HMPR $H_{2}^{\prime}$ and complete consistent MPR $R_{2}$ are obtained:

$$
H_{2}^{\prime}=\left(\begin{array}{cccc}
\{1\} & \left\{\frac{3}{7}\right\} & \left\{\frac{3}{7}, \frac{3}{2}\right\} & \{1\} \\
\left\{\frac{7}{3}\right\} & \{1\} & \left\{\frac{3}{7}, \frac{3}{2}\right\} & \left\{\frac{7}{3}\right\} \\
\left\{\frac{2}{3}, \frac{7}{3}\right\} & \left\{\frac{2}{3}, \frac{7}{3}\right\} & \{1\} & \left\{\frac{2}{3}, \frac{7}{3}\right\} \\
\{1\} & \left\{\frac{3}{7}\right\} & \left\{\frac{3}{7}, \frac{3}{2}\right\} & \{1\}
\end{array}\right)
$$


TABLE II

CONSISTENCY ASCERTAINING FOR EXAMPLE 2

\begin{tabular}{|c|c|c|c|c|c|c|}
\hline preferences & $i$ & $j$ & $k$ & $h_{i k}^{-} h_{k j}^{-}$ & $h_{i k}^{+} h_{k j}^{+}$ & Consistency test \\
\hline \multirow{3}{*}{$\bar{h}_{12}$} & 1 & 2 & 1 & 3 & 3 & $\max \left\{h^{-} h^{-} h^{-}\right\}=\frac{3}{3}$ \\
\hline & 1 & 2 & 1 & $\begin{array}{l}\overline{7} \\
2\end{array}$ & $\begin{array}{l}\overline{7} \\
7\end{array}$ & $\max \left\{h_{i j}, h_{i k} h_{k j}\right\}=\overline{7}$ \\
\hline & 1 & 2 & 3 & $\frac{2}{7}$ & $\frac{1}{2}$ & $\min \left\{h_{i j}^{+}, h_{i k}^{+} h_{k j}^{+}\right\}=\frac{-}{7}$ \\
\hline \multirow{5}{*}{$\bar{h}_{13}$} & & & & 3 & 3 & 3 \\
\hline & 1 & 3 & 1 & $\overline{7}$ & $\overline{2}$ & $\max \left\{h_{i j}^{-}, h_{i k}^{-} h_{k j}^{-}\right\}=\overline{7}$ \\
\hline & 1 & 3 & 2 & $\frac{9}{40}$ & $\frac{9}{19}$ & $\min \left\{h_{i j}^{+}, h_{i k}^{+} h_{k j}^{+}\right\}=\frac{9}{1}$ \\
\hline & & & & $\begin{array}{c}49 \\
3\end{array}$ & $\begin{array}{l}14 \\
3\end{array}$ & \\
\hline & 1 & 3 & 4 & $\overline{7}$ & $\frac{1}{2}$ & passed \\
\hline \multirow[t]{3}{*}{$\bar{h}_{14}$} & 1 & 4 & 1 & 1 & 1 & $\max \left\{h_{i j}^{-}, h_{i k}^{-} h_{k j}^{-}\right\}=1$ \\
\hline & 1 & 4 & 3 & 2 & $\frac{7}{-}$ & $\min \left\{h_{i j}^{+}, h_{i k}^{+} h_{k j}^{+}\right\}=1$ \\
\hline & & & & 7 & 2 & passed \\
\hline \multirow{3}{*}{$\bar{h}_{23}$} & 2 & 3 & 1 & 1 & $\underline{7}$ & $\max \left\{h_{i j}^{-}, h_{i k}^{-} h_{k j}^{-}\right\}=1$ \\
\hline & & & & 3 & 3 & \\
\hline & 2 & 3 & 2 & $\overline{7}$ & $\overline{2}$ & $\underset{\text { passed }}{\min \left\{h_{i j}^{+}, h_{i k}^{+} h_{k j}^{+}\right\}}=\frac{-}{2}$ \\
\hline \multirow[t]{3}{*}{$\bar{h}_{34}$} & 3 & 4 & 1 & $\frac{2}{2}$ & $\frac{7}{2}$ & $\max \left\{h_{i j}^{-}, h_{i k}^{-} h_{k j}^{-}\right\}=\frac{2}{2}$ \\
\hline & & & & $\begin{array}{l}3 \\
2\end{array}$ & $\begin{array}{l}3 \\
7\end{array}$ & \\
\hline & 3 & 4 & 3 & $\overline{3}$ & $\overline{3}$ & $\min \left\{h_{i j}^{\top}, h_{i k}^{\top} h_{k j}^{\top}\right\}=\overline{3}$ \\
\hline
\end{tabular}

and

$$
R_{2}=\left(\begin{array}{cccc}
1 & \frac{3}{7} & \frac{3}{7} & 1 \\
\frac{7}{3} & 1 & 1 & \frac{7}{3} \\
\frac{7}{3} & 1 & 1 & \frac{7}{3} \\
1 & \frac{3}{7} & \frac{3}{7} & 1
\end{array}\right)
$$

After estimating the missing values, an incomplete HMPR is converted into a complete HMPR, and Algorithm 1 can be used to generate the priority weights of alternatives. The consistency improving method proposed in Section V can be applied to incomplete HMPRs found to be inconsistent with both models (M-7) and (M-8).

\section{ILluSTRATIVE EXAMPLES AND COMPARATIVE ANALYSIS}

\section{A. Illustrative Examples}

This section offers three examples that complement the theoretical effectiveness of the approaches presented in previous sections: Examples 3 and 4 concern with $\mathrm{CMC}$ and WMC HMPRs, respectively, while Example 5 verifies the practical value of our proposal.

Example 3: Consider the following HMPR on $X=$ $\left\{x_{1}, x_{2}, x_{3}\right\}$ (adapted from Zhang [26]):

$$
H_{3}=\left(\begin{array}{ccc}
\{1\} & \left\{\frac{1}{7}, \frac{1}{5}, \frac{1}{4}, \frac{1}{3}\right\} & \left\{\frac{1}{6}, \frac{1}{3}, 1\right\} \\
\{3,4,5,7\} & \{1\} & \{2,3,5\} \\
\{1,3,6\} & \left\{\frac{1}{5}, \frac{1}{3}, \frac{1}{2}\right\} & \{1\}
\end{array}\right) .
$$

Step 1: Solving model (M-2) gives $J_{2}=0$. Thus, $H_{3}$ is CMC. Meanwhile, the following complete consistent MPR is derived:

$$
R=\left(\begin{array}{ccc}
1 & \frac{1}{3} & 1 \\
3 & 1 & 3 \\
1 & \frac{1}{3} & 1
\end{array}\right)
$$

\begin{tabular}{|c|c|c|c|c|c|c|}
\hline preferences & $i$ & $j$ & $k$ & $h_{i k}^{-} h_{k j}^{-}$ & $h_{i k}^{+} h_{k j}^{+}$ & Consistency test \\
\hline \multirow{4}{*}{$\bar{h}_{12}$} & 1 & 2 & 1 & $\frac{2}{-}$ & $\frac{5}{-}$ & $\max \left\{h_{i,}^{-} h_{\bar{j}}^{-} h_{l i}^{-}\right\}=\frac{2}{2}$ \\
\hline & & & 1 & $\overline{3}$ & $\overline{2}$ & $\max \left\{h_{i j}, n_{i k} n_{k j}\right\}=\overline{3}$ \\
\hline & 1 & 2 & 3 & $\frac{1}{4}$ & 7 & $\min \left\{h_{i j}^{+}, h_{i k}^{+} h_{k j}^{+}\right\}=\frac{J}{2}$ \\
\hline & 1 & 2 & 4 & $\frac{2}{15}$ & $\frac{3}{2}$ & passed \\
\hline \multirow{4}{*}{$\bar{h}_{13}$} & & & & $\frac{15}{3}$ & $\frac{2}{7}$ & \\
\hline & 1 & 3 & 1 & $\overline{4}$ & $\overline{2}$ & $\max \left\{h_{i j}^{-}, h_{i k}^{-} h_{k j}^{-}\right\}=\frac{-}{4}$ \\
\hline & 1 & 3 & 2 & $\frac{1}{2}$ & $\frac{15}{2}$ & $\min \left\{h_{i j}^{+}, h_{i k}^{+} h_{k j}^{+}\right\}=\frac{7}{2}$ \\
\hline & 1 & 3 & 4 & 1 & $\underline{9}$ & nassed \\
\hline \multirow{5}{*}{$\bar{h}_{14}$} & & & & $\frac{3}{1}$ & $\frac{2}{3}$ & \\
\hline & 1 & 4 & 1 & $\frac{1}{3}$ & $\overline{2}$ & $\max \left\{h_{i j}^{-}, h_{i k}^{-} h_{k j}^{-}\right\}=\frac{-}{3}$ \\
\hline & 1 & 4 & 2 & 2 & 25 & $\min \left\{h^{+} h^{+} h_{1}^{+}\right\}=-3$ \\
\hline & & & & $\begin{array}{l}3 \\
1\end{array}$ & $\begin{array}{l}4 \\
7\end{array}$ & 2 \\
\hline & 1 & 4 & 3 & $\frac{1}{4}$ & $\frac{\overline{2}}{2} \cdot \mathrm{r} \cdot \mathrm{r} \cdot \mathrm{r}$ & passed \\
\hline \multirow{4}{*}{$\bar{h}_{23}$} & 2 & 3 & 1 & 3 & 21 & $\max \left\{h_{i j}^{-}, h_{i k}^{-} h_{k j}^{-}\right\}=1$ \\
\hline & & & & $\begin{array}{c}10 \\
1\end{array}$ & 4 & \\
\hline & 2 & 3 & 2 & $\overline{2}$ & 3 & $\min \left\{h_{i j}^{+}, h_{i k}^{+} h_{k j}^{+}\right\}=3$ \\
\hline & 2 & 3 & 4 & 1 & $\frac{15}{2}$ & passed \\
\hline \multirow[t]{3}{*}{$\bar{h}_{24}$} & 2 & 4 & 1 & $\frac{2}{15}$ & $\frac{9}{4}$ & $\max \left\{h_{i j}^{-}, h_{i k}^{-} h_{k j}^{-}\right\}=1$ \\
\hline & 2 & 4 & 2 & 1 & $\frac{5}{2}$ & $\min \left\{h_{i j}^{+}, h_{i k}^{+} h_{k j}^{+}\right\}=\frac{9}{4}$ \\
\hline & 2 & 4 & 3 & $\frac{1}{6}$ & 3 & passed \\
\hline \multirow{4}{*}{$\bar{h}_{34}$} & 3 & 4 & 1 & 2 & 2 & $\max \left\{h^{-} h^{-} h_{1}^{-}\right\}=\frac{1}{1}$ \\
\hline & & & & $\begin{array}{c}21 \\
1\end{array}$ & & \\
\hline & 3 & 4 & 2 & $\frac{1}{3}$ & 5 & $\min \left\{h_{i j}^{+}, h_{i k}^{+} h_{k j}^{+}\right\}=1$ \\
\hline & 3 & 4 & 3 & $\frac{1}{2}$ & 1 & passed \\
\hline
\end{tabular}

TABLE III

CONSISTENCY ASCERTAINING FOR EXAMPLE 4

Step 2: From (31), the following priority weight vector of alternatives is obtained: $w=(0.2,0.6,0.2)^{T}$, and the alternatives ranking would be: $x_{2} \succ x_{1} \sim x_{3}$.

Example 4: Consider the following HMPR (adapted from Lin and Wang [19]):

$$
H_{4}=\left(\begin{array}{cccc}
\{1\} & \left\{\frac{2}{3}, 2, \frac{5}{2}\right\} & \left\{\frac{3}{4}, \frac{5}{2}, \frac{7}{2}\right\} & \left\{\frac{1}{3}, \frac{3}{2}\right\} \\
\left\{\frac{2}{5}, \frac{1}{2}, \frac{3}{2}\right\} & \{1\} & \left\{\frac{1}{2}, 2,3\right\} & \left\{1,2, \frac{5}{2}\right\} \\
\left.\frac{2}{7}, \frac{2}{5}, \frac{4}{3}\right\} & \left\{\frac{1}{3}, \frac{1}{2}, 2\right\} & \{1\} & \left\{\frac{1}{3}, \frac{1}{2}, 1\right\} \\
\left\{\frac{2}{3}, 3\right\} & \left\{\frac{2}{5}, \frac{1}{2}, 1\right\} & \{1,2,3\} & \{1\}
\end{array}\right) .
$$

Step 1: Solving model (M-2), we have $J_{2}=0.5754$, which means that HMPR $H_{4}$ is not CMC.

Step 2: Expression (17) is used to check the weakly multiplicative consistency property for $H_{4}$, with the corresponding processes shown in Table III. It is concluded that HMPR $H_{4}$ is WMC. Notice that this could have been done using (22). Indeed, HMPR $H_{4}$ is WMC because

$$
\begin{gathered}
\bigcap_{k=1}^{4}\left[h_{1 k}^{-} h_{k 2}^{-}, h_{1 k}^{+} h_{k 2}^{+}\right]= \\
\left.\qquad \frac{2}{3}, \frac{5}{2}\right] \bigcap\left[\frac{2}{3}, \frac{5}{2}\right] \bigcap\left[\frac{1}{4}, 7\right] \\
\bigcap\left[\frac{2}{15}, \frac{3}{2}\right] \neq \varnothing
\end{gathered}
$$




$$
\begin{aligned}
\bigcap_{k=1}^{4}\left[h_{1 k}^{-} h_{k 3}^{-}, h_{1 k}^{+} h_{k 3}^{+}\right]= & {\left[\frac{3}{4}, \frac{7}{2}\right] \bigcap\left[\frac{1}{3}, \frac{15}{2}\right] \bigcap\left[\frac{3}{4}, \frac{7}{2}\right] } \\
& \bigcap\left[\frac{1}{3}, \frac{9}{2}\right] \neq \varnothing \\
\bigcap_{k=1}^{4}\left[h_{1 k}^{-} h_{k 4}^{-}, h_{1 k}^{+} h_{k 4}^{+}\right]= & {\left[\frac{1}{3}, \frac{3}{2}\right] \bigcap\left[\frac{2}{3}, \frac{25}{4}\right] \bigcap\left[\frac{1}{4}, \frac{7}{2}\right] } \\
& \bigcap\left[\frac{1}{3}, \frac{3}{2}\right] \neq \varnothing \\
\bigcap_{k=1}^{4}\left[h_{2 k}^{-} h_{k 3}^{-}, h_{2 k}^{+} h_{k 3}^{+}\right]= & {\left[\frac{3}{10}, \frac{21}{4}\right] \bigcap\left[\frac{1}{2}, 3\right] \bigcap\left[\frac{1}{2}, 3\right] } \\
\bigcap_{k=1}^{4}\left[h_{2 k}^{-} h_{k 4}^{-}, h_{2 k}^{+} h_{k 4}^{+}\right]= & {\left[\frac{2}{15}, \frac{9}{4}\right] \bigcap\left[1, \frac{5}{2}\right] \bigcap\left[1, \frac{5}{2}\right] } \\
& \bigcap\left[\frac{1}{6}, 3\right] \neq \varnothing \\
\stackrel{4}{4}\left[h_{3 k}^{-} h_{k 4}^{-}, h_{3 k}^{+} h_{k 4}^{+}\right]= & {\left[\frac{2}{21}, 2\right] \bigcap\left[\frac{1}{3}, 5\right] \bigcap\left[\frac{1}{3}, 1\right] } \\
k_{k=1} & \bigcap\left[\frac{1}{3}, 1\right] \neq \varnothing .
\end{aligned}
$$

Since HMPR $H_{4}$ is WMC, the priority weights of alternatives are derived by solving models (M-3)-(M-5).

Step 3: Solving model (M-3) gives $J_{3}=0$, and $w=$ $(0.25,0.25,0.25,0.25)^{T}$. From (3), we obtain the following complete consistent MPR:

$$
R=\left(\begin{array}{llll}
1 & 1 & 1 & 1 \\
1 & 1 & 1 & 1 \\
1 & 1 & 1 & 1 \\
1 & 1 & 1 & 1
\end{array}\right)
$$

Solving models (M-4) and (M-5), the following interval priority vector of alternatives is obtained from $H_{4}: \quad w\left(H_{4}\right)=([0.1875,0.3818], \quad[0.2222,0.4091]$, $[0.0952,0.2667], \quad[0.1739,0.3333]) . \quad$ Using expression (32), the ranking of the alternatives would be: $\begin{array}{lll}0.5813 & 0.5878 \quad 0.7196\end{array}$

$x_{2} \succ x_{1} \succ x_{4} \succ x_{3}$. Thus, alternative $x_{2}$ is superior to alternative $x_{1}$ with $58.13 \%$ possibility degree, alternative $x_{1}$ is superior to alternative $x_{4}$ with $58.78 \%$ possibility degree, while alternative $x_{4}$ is superior alternative $x_{3}$ with $71.96 \%$ possibility degree.

Example 5: A practical problem is considered where an investment company is looking to invest a sum of money in the best of the following four possible investment options.

1) $x_{1}$ is an energy company.

2) $x_{2}$ is a medical corporation.

3) $x_{3}$ is a high-tech company.

4) $x_{4}$ is a food company.

The investment company evaluates the four alternative companies with the help of a third-party evaluation agency, which provides the following HMPR information:

$$
H_{5}=\left(\begin{array}{cccc}
\{1\} & \{3\} & \{5,7\} & \{3\} \\
\left\{\frac{1}{3}\right\} & \{1\} & \left\{\frac{1}{9}, \frac{1}{7}\right\} & \{5\} \\
\left\{\frac{1}{7}, \frac{1}{5}\right\} & \{7,9\} & \{1\} & \left\{\frac{1}{7}, \frac{1}{5}\right\} \\
\left\{\frac{1}{3}\right\} & \left\{\frac{1}{5}\right\} & \{5,7\} & \{1\}
\end{array}\right) .
$$

Step 1: Solving model (M-2) gives $J_{2}=10.3296$. Thus, $H_{5}$ is not CMC.

Step 2: Solving model (M-3) gives $J_{3}=5.9238$. Thus, $H_{5}$ is not WMC. This means that we are in the presence of an inconsistent HMPR.

Step 3: The optimal deviation values are $d_{23}^{+}=1.5238$, $d_{34}^{+}=0.4$, and $d_{24}^{-}=4$; so, $h_{23}, h_{24}$, and $h_{34}$ are the inconsistent elements of HMPR $H_{5}$. From (29), the adjusted elements are $\bar{h}_{23}=\{1 / 9,1.667\}, \bar{h}_{24}=\{1\}$, and $\bar{h}_{34}=\{1 / 7,3 / 5\}$. From (30), the improved HMPR $\mathrm{H}_{5}$ is

$$
\bar{H}_{5}=\left(\begin{array}{cccc}
\{1\} & \{3\} & \{5,7\} & \{3\} \\
\left\{\frac{1}{3}\right\} & \{1\} & \left\{\frac{1}{9}, 1.667\right\} & \{1\} \\
\left\{\frac{1}{7}, \frac{1}{5}\right\} & \{0.6,9\} & \{1\} & \left\{\frac{1}{7}, \frac{3}{5}\right\} \\
\left\{\frac{1}{3}\right\} & \{1\} & \left\{\frac{5}{3}, 7\right\} & \{1\}
\end{array}\right) .
$$

Step 4: Solving model (M-2) implies that $\bar{H}_{5}$ is not CMC. Step 5: Solving model $(\mathrm{M}-3)$ gives $J_{3}=0$. Thus, $\bar{H}_{5}$ is WMC.

Step 6: Solving models (M-4) and (M-5), the interval priority weight vector of the alternatives for $\bar{H}_{5}$ is $w\left(\bar{H}_{5}\right)=([0.5357,0.5382], \quad[0.1786,0.1794]$, [0.1029, 0.1071], [0.1786, 0.1794]). Applying (32) results in the following ranking of the four alternatives: $x_{1}>x_{2} \sim x_{4} \succ x_{3}$. This means that investment options $x_{1}$ is superior to investment options $x_{2}$ with $100 \%$ possibility degree, investment options $x_{2}$ is equally preferred to investment options $x_{4}$, and investment options $x_{4}$ is superior to investment options $x_{3}$ with $100 \%$ possibility degree. Therefore, the optimal investment would be $x_{1}$.

In decision-making problems, the consistency problem is closely related to the reliability of preferences provided by decision makers. The rationality of the judgments determines the reliability of the final decision result. It is worth noting that the consistency improvement of HMPRs in this process plays a role in regulating the logic and rationality of the given preference information. Therefore, in practice, our proposal contributes to achieving reliable decision-making results.

In what follows, a discussion and a simulation analysis are reported to illustrate the availability and advantages of the proposed method.

\section{B. Discussion, Simulation, and Comparative Analysis}

In this section, we compare the peculiarities of existing methods and discuss the advantages of our proposed methods. A summary of the improvements of the proposed method based on the previous illustrative examples is provided. In addition, a systematic analysis with the help of simulation experiments is carried out, which clearly and intuitively highlights the superior performance of the proposed method. 
1) Discussion: In view of the evident differences with the existing consistency studies, the proposed approach improvements can be summarized as follows.

1) As far as we are aware, the proposed approach is the first attempt to study simultaneously both completely multiplicative consistency and weakly multiplicative consistency properties for HMPRs, which represents a more effective and precise way to describe and detect consistency. Meng et al. [25] studied the multiplicative consistency property of HMPRs. They proposed a strict consistency concept for HMPRs that requires the existence of multiplicative consistent MPRs for every value in every HMEs. This means that in practice most HMPRs will fail to verify Meng et al.'s [25] definition of consistency property. For example, $\mathrm{H}_{3}$ (Example 3), which was judged to be CMC, does not satisfy Meng et al.'s consistency definition. Although theoretically there exist HMPRs that verify Meng et al.'s consistency definition, this is not reasonable in practice. Hesitancy means that a decision maker is unsure about the preference values when comparing two alternatives, though he/she can give some possible preference values (hence the hesitation). If for every value in every HME, a consistent MPR exists, then this would imply a level of consistency knowledge by the decision maker that would make hesitancy improbable and therefore impractical in a hesitancy environment. As the decision maker is hesitant, we should aim to find the reasonable information (i.e., consistent information) from his/her hesitant information, which is exactly the aim of the proposed method.

2) Zhang and $\mathrm{Wu}$ proposed two consistency improvement methods in [17] and [21]. These methods rely on a $\beta$ normalization process, which converts the HMEs so that they all have the same number of values, and then the HMPR is managed as several MPRs. The normalization process obviously distorts the DM's original information and the results obtained could be unrelated to the original information, which makes them unreliable. In this article, the proposed method does not rely on any normalization process, which translates into minimal changes of the original information of DMs and lower computational cost. Moreover, Xu et al. [46] pointed out that Zhang and Wu's [21] consistency process is artificial, the consistent HMPR may not be an HMPR because the improved MPRs will not be arranged in ascending order. Additionally, the smaller the improvement process consistency threshold in [21] is, the larger the number of iterations and the computational cost are.

3) The priority weights of alternatives derived from the proposed method are of interval nature. As the DM's information is hesitant, it is more logical and natural to derive interval weights from consistent HMPRs than exact priority weights as proposed by Zhu and $\mathrm{Xu}$ [18]. Although Zhang and Wu's [17] weight-derivation algorithm for HMPRs results in an interval priority weight vector for $H_{5}, w=([0.3938,0.4581],[0.1715,0.1729]$, [0.1882, 0.2108], [0.1823, 0.2225]), which leads to the
TABLE IV

Comparison Between the Existing STUdies ANd Our Proposal

\begin{tabular}{|c|c|c|c|c|c|}
\hline & $\begin{array}{l}\text { The } \\
\text { proposed } \\
\text { method }\end{array}$ & $\begin{array}{c}\text { Zhang } \\
\text { and } \mathrm{Wu} \\
{[21]}\end{array}$ & $\begin{array}{l}\text { Meng } \\
\text { et al. } \\
{[25]}\end{array}$ & $\begin{array}{c}\text { Zhang } \\
\text { and Wu } \\
{[17]}\end{array}$ & $\begin{array}{c}\text { Sahu and } \\
\text { Gupta } \\
{[22]}\end{array}$ \\
\hline $\begin{array}{l}\text { Ascertain } \\
\text { consistency }\end{array}$ & $\checkmark$ & $\checkmark$ & $\checkmark$ & $\checkmark$ & $\checkmark$ \\
\hline $\begin{array}{l}\text { Consistency } \\
\text { thresholds }\end{array}$ & $x$ & $\checkmark$ & $x$ & $x$ & $\checkmark$ \\
\hline $\begin{array}{c}\alpha \text { or } \beta \\
\text { normalization }\end{array}$ & $x$ & $\checkmark$ & $x$ & $\checkmark$ & $\checkmark$ \\
\hline $\begin{array}{c}\text { Repair } \\
\text { inconsistency } \\
\end{array}$ & $\checkmark$ & $\checkmark$ & $x$ & $\checkmark$ & $x$ \\
\hline $\begin{array}{c}\text { Minimal } \\
\text { deviation from } \\
\text { decision- } \\
\text { maker's original } \\
\text { judgments } \\
\end{array}$ & $\checkmark$ & $x$ & $x$ & $x$ & $x$ \\
\hline $\begin{array}{l}\text { Ability to } \\
\text { maintain } \\
\text { decision- } \\
\text { makers' } \\
\text { hesitation in } \\
\text { weights } \\
\text { derivation } \\
\end{array}$ & $\checkmark$ & $x$ & $\checkmark$ & $\checkmark$ & $x$ \\
\hline $\begin{array}{c}\text { Ability to } \\
\text { address } \\
\text { incomplete } \\
\text { HMPRs }\end{array}$ & $\checkmark$ & $x$ & $\checkmark$ & $x$ & $\checkmark$ \\
\hline $\begin{array}{c}\text { Acceptable } \\
\text { computational } \\
\text { complexity }\end{array}$ & $\checkmark$ & - & - & - & - \\
\hline
\end{tabular}

optimal choice $x_{1}$, which is consistent with the proposed approach, although it is based on an additional normalization process, which implies higher computational cost.

4) The proposed approach can be utilized to solve decisionmaking problems with incomplete HMPRs via the two multiplicative consistency goal programming models developed to ascertain the consistency property and to estimate the missing values. The existent literature method by Sahu and Gupta [22] requires a normalization process to improve the consistency, and therefore is subjected to the previously mentioned drawbacks. Thus, the proposed approach can deal with incomplete information in HMPR, which allows DMs or decision organizations to express their preferences more flexibly, and therefore more effectively.

In summary, the above analysis shows that the performance of the proposal approach can compete with other approaches. The comparative analysis, based on eight performance criteria, of these methods is summarized in Table IV. The label " $\sqrt{ }$ " means that the method is very suitable, "-" means that the method is acceptable, while " $x$ " means that the method performs poorly on the given criterion.

From Table IV, it can see that the functionality of the proposed approach is powerful, and that it can help to 1) determine the consistency type without the help of consistency threshold setting and normalization process; 2) repair inconsistency with lower information distortion and computation; 3) derive interval weights based on the decision maker's hesitation; and 4) solve decision-making problems with incomplete HMPRs. Consequently, the proposed approach can deal 
with decision-making problems with HMPRs more flexibly, reasonably, and effectively.

2) Simulation and Comparative Analysis: In order to further show the effectiveness and advantages of the proposed method, Monte Carlo simulation experiments are carried out and analyzed. Further, the proposed method is compared with the methods by Zhang and $\mathrm{Wu}$ [17] and Zhang and $\mathrm{Wu}$ [21], since their methods also proposed different consistency concepts and consistency improving processes. The $\beta$-normalization method is used in [17] and [21] with $\bar{h}_{i j}=$ $\left(h_{i j}^{+}\right)^{\varsigma} \times\left(h_{i j}^{-}\right)^{(1-\varsigma)}$ used to add some values to the HMEs of shorter length to make all the HMEs have the same length. In this article, we assume $\varsigma=0.5$. Both Zhang and $\mathrm{Wu}$ [17] and Zhang and $\mathrm{Wu}$ [21] splited the HMPR into several MPRs. Zhang and $\mathrm{Wu}[17]$ used Saaty's consistency ratio $(\mathrm{CR}<0.1)$ to check whether these MPRs are of acceptable consistency. If any of the MPR is not acceptable consistent, Xu and Wei [51]'s Algorithm I (with $\lambda=0.5$ ) is used to improving its consistency. Notice that Zhang and $\mathrm{Wu}$ [21] proposed another algorithm (referred to as [21, Algorithm 2]) to improve consistency. In the method, a consistency threshold $\overline{\mathrm{CI}}$ is set in advance $(\overline{\mathrm{CI}}=1.01)$. Meng et al.'s [25] method only find the consistent MPRs in an HMPR, with no method to repair the inconsistency proposed when there is no such consistent MPR in an HMPR. Sahu and Gupta [22] proposed a method to estimate the missing values in an incomplete HMPR, and Zhang and Wu's [17] is adopted to check whether the complete HMPR is of acceptable consistency. If the complete HMPR is not consistent, no consistency improving method is provided. Therefore, in the following, we only do simulations and compare the proposed method with Zhang and $\mathrm{Wu}$ [17] and Zhang and $\mathrm{Wu}[21]$ 's methods.

A total of 1000 HMPRs with different dimensions, ranging from 3 to 9, are randomly generated. In order to be close to the actual decision-making scenario, we assume that the number of elements in each HME is less than 3 . Furthermore, all the randomly generated values are in Saaty's scale $\{1 / 9,1 / 8, \ldots, 1 / 2,1, \ldots, 9\}$. In order to compare the performances of the different methods, we propose the following criteria.

1) Length Change Ratio:

$$
\mathrm{LCR}=\frac{2 \sum_{i=1}^{n-1} \sum_{j=i+1}^{n} \bar{f}_{i j}}{n(n-1)}
$$

where $\bar{f}_{i j}=\left\{\begin{array}{ll}0, & l_{h_{i j}^{(0)}}=l_{h_{i j}^{*}} \\ 1, & \text { otherwise }\end{array}\right.$ denotes whether the length of an HME is changed; and $H^{(0)}=\left(h_{i j}^{(0)}\right)$ and $H^{*}=$ $\left(h_{i j}^{*}\right)$ are the original and the final adjusted HMPRs, respectively.

2) Numerical Adjustment Ratio:

$$
\mathrm{NAR}=\frac{2 \sum_{i=1}^{n-1} \sum_{j=i+1}^{n} f_{i j}}{n(n-1)}
$$

where $f_{i j}=\left\{\begin{array}{ll}0, & h_{i j}^{(0)}=h_{i j}^{*} \\ 1, & \text { otherwise }\end{array}\right.$ denotes whether the values in an HME $h_{i j}^{(0)}$ are adjusted.
TABLE V

AVERAGE LCR, NAR, AD, LAD, DR, AND ITERATION VALUES OF

\begin{tabular}{|c|c|c|c|c|c|c|c|}
\hline$n$ & methods & LCR & NAR & $\mathrm{AD}$ & LAD & DR & Iterations \\
\hline \multirow[t]{3}{*}{3} & This paper & 0 & 0.149 & 0.122 & 0.440 & 1.227 & 1 \\
\hline & Zhang and $\mathrm{Wu}$ [21] & 0.4863 & 1 & 3.867 & 1.908 & 2.076 & 2.973 \\
\hline & Zhang and $\mathrm{Wu}$ [17] & 0.4863 & 1 & 2.703 & 1.319 & 1.726 & 3.078 \\
\hline \multirow[t]{3}{*}{4} & This paper & 0 & 0.301 & 0.283 & 0.986 & 1.4223 & 1 \\
\hline & Zhang and $\mathrm{Wu}[21]$ & 0.6213 & 1 & 4.685 & 2.967 & 2.3226 & 3.033 \\
\hline & Zhang and $\mathrm{Wu}$ [17] & 0.6213 & 1 & 3.965 & 1.992 & 2.0718 & 4.557 \\
\hline \multirow[t]{3}{*}{5} & This paper & 0 & 0.436 & 0.407 & 1.46 & 1.603 & 1 \\
\hline & Zhang and $\mathrm{Wu}[21]$ & 0.6565 & 1 & 5.013 & 3.599 & 2.5201 & 3.051 \\
\hline & Zhang and $\mathrm{Wu}[17]$ & 0.6565 & 1 & 4.477 & 2.504 & 2.2276 & 5.257 \\
\hline \multirow[t]{3}{*}{6} & This paper & 0 & 0.518 & 0.524 & 1.848 & 1.743 & 1 \\
\hline & Zhang and $\mathrm{Wu}[21]$ & 0.6684 & 1 & 5.370 & 4.098 & 2.7102 & 3.078 \\
\hline & Zhang and $\mathrm{Wu}[17]$ & 0.6684 & 1 & 4.928 & 2.913 & 2.4092 & 5.652 \\
\hline \multirow[t]{3}{*}{7} & This paper & 0 & 0.636 & 0.603 & 1.963 & 1.7717 & 1 \\
\hline & Zhang and $\mathrm{Wu}[21]$ & 0.6606 & 1 & 5.552 & 4.369 & 2.8243 & 3.123 \\
\hline & Zhang and $\mathrm{Wu}[17]$ & 0.6606 & 1 & 5.114 & 3.120 & 2.5068 & 5.848 \\
\hline \multirow[t]{3}{*}{8} & This paper & 0 & 0.836 & 0.699 & 2.464 & 1.9121 & 1 \\
\hline & Zhang and $\mathrm{Wu}[21]$ & 0.6669 & 1 & 5.654 & 4.611 & 2.9306 & 3.153 \\
\hline & Zhang and $\mathrm{Wu}[17]$ & 0.6669 & 1 & 5.212 & 3.294 & 2.5901 & 5.939 \\
\hline \multirow[t]{3}{*}{9} & This paper & 0 & 0.991 & 0.775 & 2.888 & 2.0213 & 1 \\
\hline & Zhang and $\mathrm{Wu}[21]$ & 0.6661 & 1 & 5.740 & 4.779 & 3.0114 & 3.2580 \\
\hline & Zhang and $\mathrm{Wu}$ [17] & 0.6661 & 1 & 5.265 & 3.377 & 2.6375 & 5.972 \\
\hline
\end{tabular}
DIFFERENT METHODS

3) Absolute Deviation:

$$
A D=\frac{2}{n(n-1)} \sum_{i=1}^{n-1} \sum_{j=i+1}^{n}\left|h_{i j}^{(0)}-h_{i j}^{*}\right|
$$

Absolute deviation (AD) measures the average numerical difference between the original HMPR $H^{(0)}$ and the final improved HMPR $H^{*}$. Since the lengths in each $h_{i j}$ between $H^{(0)}$ and $H^{*}$ are different in Zhang and $\mathrm{Wu}$ [17] and Zhang and $\mathrm{Wu}[21]$, the $\beta$-normalization HMPR is used on the original HMPR to compute $\mathrm{AD}$ for the proposed approach.

4) Logarithm Absolute Deviation:

$$
\mathrm{LAD}=\frac{2}{n(n-1)} \sum_{i=1}^{n-1} \sum_{j=i+1}^{n}\left(\ln \left(h_{i j}^{(0)}\right)-\ln \left(h_{i j}^{*}\right)\right)^{2} .
$$

5) Difference Ratio: Li et al. [52] introduced a ratio-based concept to gauge the difference between two interval multiplicative comparison matrices. Based on this idea, the below difference ratio (DR) is proposed to measure the difference between the original HMPR and the improved HMPR

$$
\begin{aligned}
\operatorname{DR}\left(H^{(0)}, H^{*}\right)= & \left(\prod_{i<j}\left(\frac{\max \left\{h_{i j}^{(0)-}, h_{i j}^{*-}\right\}}{\min \left\{h_{i j}^{(0)-}, h_{i j}^{*-}\right\}}\right)\right. \\
& \left.\times\left(\frac{\max \left\{h_{i j}^{(0)+}, h_{i j}^{*+}\right\}}{\min \left\{h_{i j}^{(0)+}, h_{i j}^{*+}\right\}}\right)\right)^{\frac{1}{n(n-1)}} .
\end{aligned}
$$

Obviously, $\operatorname{DR}\left(H^{(0)}, H^{*}\right) \geq 1$. The smaller the ratio $\mathrm{DR}\left(H^{(0)}, H^{*}\right)$, the closer $H^{(0)}$ is to $H^{*}$. In particular, if $\operatorname{DR}\left(H^{(0)}, H^{*}\right)=1, H^{(0)}=H^{*}$.

Table $\mathrm{V}$ lists the average values of length change ratio (LCR), numerical adjustment ratio (NAR), $\mathrm{AD}$, logarithm AD (LAD), and DR for each of the three considered methods, which are represented in Fig. 3 to help visualize the different methods' performance. 


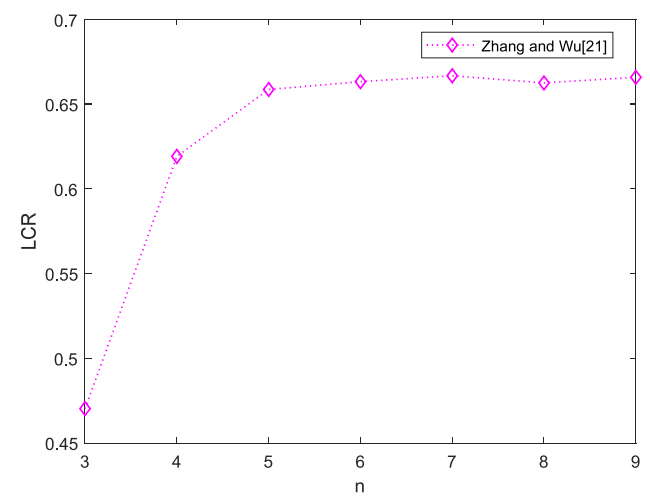

(a)

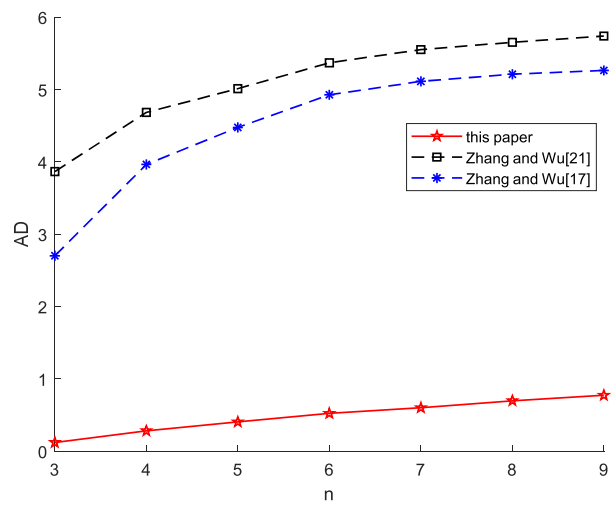

(c)

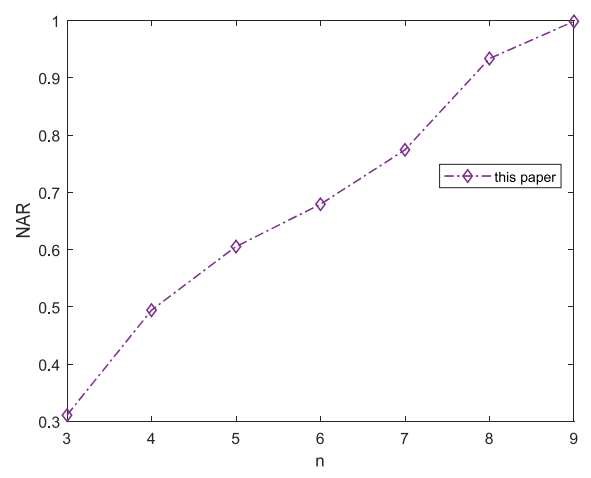

(b)

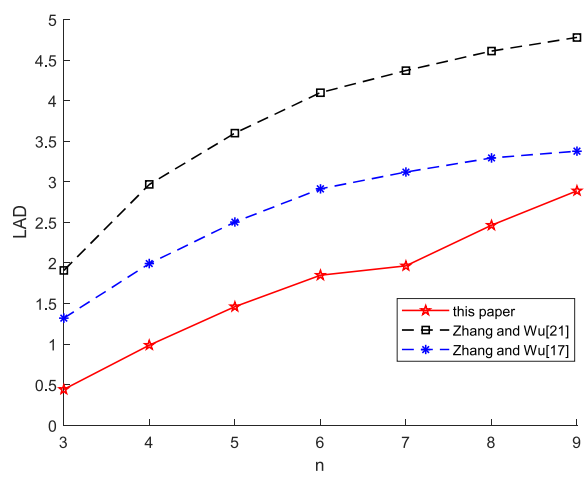

(d)

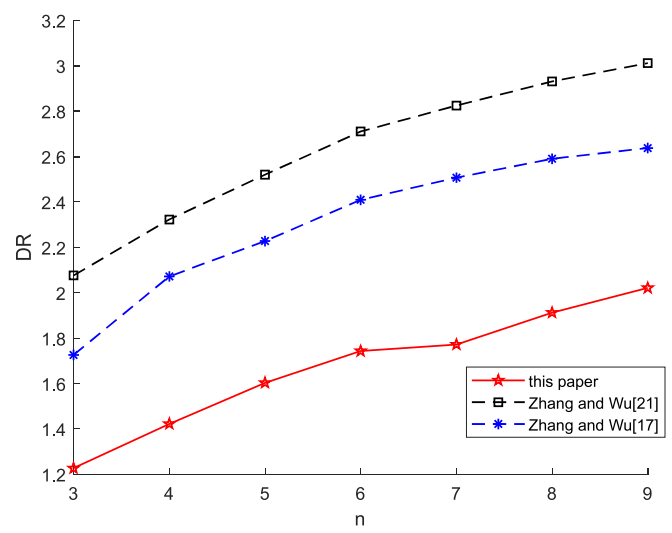

(e)

Fig. 3. (a) LCR of Zhang and Wu [17]. (b) NAR of this article. (c) AD. (d) LAD. (e) DR.

In Fig. 3(a), since the length of HMEs is not changed by the proposed method, the corresponding LCR value is always equal to 0 at every one of the considered dimensions. Zhang and $\mathrm{Wu}$ [17] and Zhang and $\mathrm{Wu}$ [21] used the same normalization methods, thus their LCR values coincide and therefore there is only need to draw the LCR values for one of them. The LCR values increase drastically from 3 to 5 , while they change little when $n$ is from 5 to 9. In Fig. 3(b), the NAR values in Zhang and Wu [17] and Zhang and Wu [21] are always equal to 1 , which means that all the values are revised in their consistency improving processes. However, the NAR values increases from $0.149(n=3)$ to $0.991(n=9)$ for the proposed method. These two indexes show that the proposed method perform best in retain the decision makers' original information as much as possible.

In Fig. 3(c)-(e), the AD, LAD, and DR values all increase with the value of $n$. However, in all cases, the proposed method results in the smallest values, with Zhang and $\mathrm{Wu}$ [21] resulting in the largest. Therefore, the proposed method produces improved consistent HMPRs closest to the original HMPRs. These results reinforce the achievement of the aim of the proposed method to retain the decision makers' original information as much as possible.

3) Computational Complexity: Regarding computational complexity as measured by the average number of iterations required to complete the overall process, again the proposed 


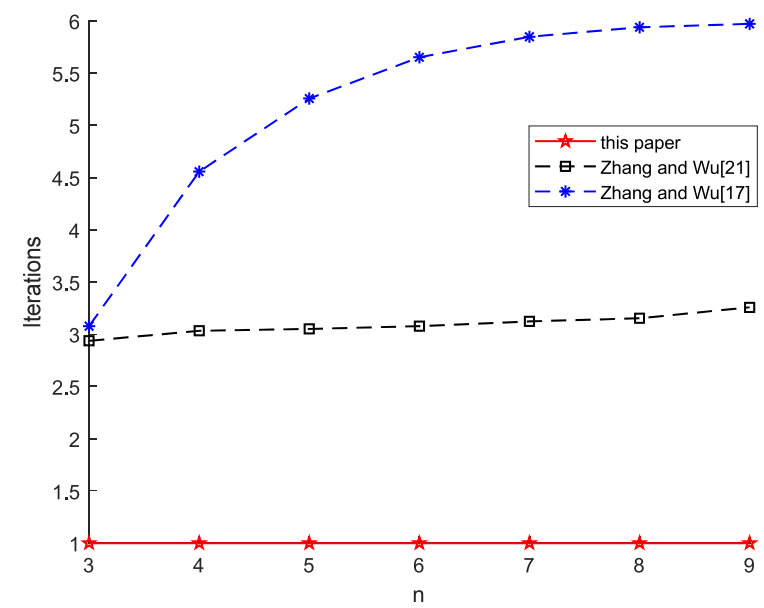

Fig. 4. Average iterations of different methods.

method is superior to the method by Zhang and Wu. This information is provided in the last column of Table $\mathrm{V}$ and depicted in Fig. 4. The proposed method requires in all cases 1 iteration to improve consistency, Zhang and Wu's [21] method is stable at three iterations on average, while Zhang and Wu's [17] method need 3 to 6 iterations on average increasing with the dimension value.

As mentioned earlier, Zhang and $\mathrm{Wu}[21]$ required that all HMEs have the same length before the process of consistency ascertaining. This normalization method, and therefore its associated complicated calculation process, is superfluous for the proposed method. Since the length of HMEs increases with the normalization process, the HMPR will be converted into a high number of MPRs to judge its consistency, which will increase the computation cost when compared to the proposed method. On the other hand, Zhang and $\mathrm{Wu}$ [21] preset a consistency threshold in the process of consistency checking and improvement. Decreasing the threshold value implies an increase of the number of iterations and, as a consequence, the computational cost will increase. In contrast, the consistency properties of HMPRs proposed in this article can directly be ascertain without the need of a normalization process or a consistency threshold, while the inconsistency repairing method only revises the inconsistent elements, and therefore most of the decision maker's judgments are unchanged. Most importantly, the proposed approach can achieve multiplicative consistency ascertaining, inconsistency repairing, and weights derivation for HMPRs in one iteration.

Meng et al. [25] also implemented the consistency test based on the decision maker's original HMPR without the normalization process. Their consistency determination and improvement process can also be completed within one iteration. However, Meng et al.'s approach requires to detect that for each value in each HME a multiplicatively consistent MPR needs to be detected. As the length of the HMEs increases, the number of multiplicatively consistent MPRs to be found increases. Namely, there are a total of $\prod_{i<j} l_{h_{i j}}$ MPRs that need to be judged, and at least the minimum of $l_{h i j}$ models to operate. Hence, this method may not be suitable to be applied in practical decision-making problems due to its high computational cost.

To summarize, compared with the existing methods, the proposed method has lowest computational complexity and cost. Therefore, the proposed method is a highly functional and computationally convenient method.

\section{CONCLUSION}

In this article, two types of multiplicative consistency of HMPRs, completely multiplicative consistency and weakly multiplicative consistency, are investigated simultaneously. A number of 0-1 mixed programming models are established to ascertain these consistency properties. The following cases are addressed.

1) If an HMPR is CMC, then the corresponding multiplicative consistent MPR can be found.

2) If an HMPR is not CMC but WMC, then interval priority weights of alternatives are derived, which allows to rank them.

3) If an HMPR is not consistent, only the inconsistent elements are revised to repair the inconsistency, which means that most of the decision maker's judgments are unchanged.

4) These models have also been extended to the case of incomplete HMPRs by estimating the missing values.

In the future, the research areas to focus on include:

1) how to apply the proposed method to other types of preference relations [53], [54];

2) in addition to the consistency analysis of individual decision makers, consensus analysis with HMPRs is also an important research topic in group decision making [55]-[59];

3) investigate new algorithms for group decision-making problems to tackle practical problems [60]-[62].

\section{REFERENCES}

[1] T. L. Saaty, The Analytic Hierarchy Process. New York, NY, USA: McGraw-Hill, 1980.

[2] E. Herrera-Viedma, S. Alonso, F. Chiclana, and F. Herrera, "A consensus model for group decision making with incomplete fuzzy preference relations," IEEE Trans. Fuzzy Syst., vol. 15, no. 5, pp. 863-877, Oct. 2007

[3] Y. J. Xu, K. W. Li, and H. M. Wang, "Consistency test and weight generation for additive interval fuzzy preference relations," Soft Comput., vol. 18, no. 8, pp. 1499-1513, 2014.

[4] S. Genç, F. E. Boran, D. Akay, and Z. S. Xu, "Interval multiplicative transitivity for consistency, missing values and priority weights of interval fuzzy preference relations," Inf. Sci., vol. 180, no. 24 , pp. 4877-4891, 2010.

[5] Z. S. Xu and J. Chen, "Some models for deriving the priority weights from interval fuzzy preference relations," Eur. J. Oper. Res., vol. 184, no. 1 , pp. 266-280, 2008.

[6] Z. S. Xu, "Intuitionistic preference relations and their application in group decision making," Inf. Sci., vol. 177, no. 11, pp. 2363-2379, 2007.

[7] Z. M. Zhang and W. Pedrycz, "Intuitionistic multiplicative group analytic hierarchy process and its use in multicriteria group decisionmaking," IEEE Trans. Cybern., vol. 48, no. 7, pp. 1950-1962, Jul. 2018.

[8] E. Szmidt and J. Kacprzyk, "A consensus-reaching process under intuitionistic fuzzy preference relations," Int. J. Intell. Syst., vol. 18, no. 7, pp. 837-852, 2003.

[9] Y. Lin and Y. M. Wang, "Group decision making with consistency of intuitionistic fuzzy preference relations under uncertainty," IEEE/CAA J. Autom. Sinica, vol. 5, no. 3, pp. 741-748, May 2018. 
[10] Y. J. Xu, F. Ma, and F. Herrera, "Revisiting inconsistent judgments for incomplete fuzzy linguistic preference relations: Algorithms to identify and rectify ordinal inconsistencies," Knowl. Based Syst., vol. 163, pp. 305-319, Jan. 2019.

[11] Y. C. Dong, Y. F. Xu, and H. Y. Li, "On consistency measures of linguistic preference relations," Eur. J. Oper. Res., vol. 189, no. 2, pp. 430-444, 2008.

[12] E. Herrera-Viedma, L. Martinez, F. Mata, and F. Chiclana, "A consensus support system model for group decision-making problems with multigranular linguistic preference relations," IEEE Trans. Fuzzy Syst., vol. 13, no. 5, pp. 644-658, Oct. 2005.

[13] S. Alonso, F. J. Cabrerizo, F. Chiclana, F. Herrera, and E. HerreraViedma, "Group decision making with incomplete fuzzy linguistic preference relations," Int. J. Intell. Syst., vol. 24, no. 2, pp. 201-222, 2009.

[14] V. Torra, "Hesitant fuzzy sets," Int. J. Intell. Syst., vol. 25, no. 6, pp. 529-539, 2010.

[15] M. M. Xia and Z. S. Xu, "Managing hesitant information in GDM problems under fuzzy and multiplicative preference relations," Int. J. Uncertainty Fuzziness Knowl. Based Syst., vol. 21, no. 6, pp. 865-897, 2013.

[16] M. Liu, W. Zhou, and Y. L. Duan, "Generalized hesitant multiplicative preference relations and the analytic risk-network process," Inf. Sci., vol. 540, pp. 345-369, Nov. 2020.

[17] Z. M. Zhang and C. Wu, "Deriving the priority weights from hesitant multiplicative preference relations in group decision making," Appl. Soft Comput., vol. 25, pp. 107-117, Dec. 2014.

[18] B. Zhu and Z. S. Xu, "Analytic hierarchy process-hesitant group decision making," Eur. J. Oper. Res., vol. 239, no. 3, pp. 794-801, 2014.

[19] Y. Lin and Y.-M. Wang, "Prioritization of hesitant multiplicative preference relations based on data envelopment analysis for group decision making," Neural Comput. Appl., vol. 31, no. 2, pp. 437-447, 2019.

[20] Y. He, Z. S. Xu, and J. Gu, "An approach to group decision making with hesitant information and its application in credit risk evaluation of enterprises," Appl. Soft Comput., vol. 43, pp. 159-169, Jun. 2016.

[21] Z. M. Zhang and C. Wu, "A decision support model for group decision making with hesitant multiplicative preference relations," Inf. Sci., vol. 282, pp. 136-166, Oct. 2014.

[22] M. Sahu and A. Gupta, "Improving the consistency of incomplete hesitant multiplicative preference relation," Opsearch, vol. 56, no. 1, pp. 324-343, 2019.

[23] Q. Mou, Z. S. Xu, H. C. Liao, and Z. N. Hao, "Two regression methods for hesitant multiplicative preference relations with different consistencies," Soft Comput., vol. 23, no. 5, pp. 7029-7044, 2018.

[24] D. M. Nie, "A method for repairing the consistency of hesitant multiplicative preference relations in group decision making," Control Eng. China, vol. 23, no. 4, pp. 623-628, 2016.

[25] F. Y. Meng, J. Tang, Q. X. An, and X. H. Chen, "A new procedure for hesitant multiplicative preference relations," Int. J. Intell. Syst., vol. 34, no. 5, pp. 819-857, 2019.

[26] Z. M. Zhang, "Some hesitant multiplicative aggregation operators and their application in group decision making with hesitant multiplicative preference relations," Int. J. Fuzzy Syst., vol. 18, no. 2, pp. 177-197, 2016.

[27] Y. He and Z. S. Xu, "A consensus reaching model for hesitant information with different preference structures," Knowl. Based Syst., vol. 135, pp. 99-112, Nov. 2017.

[28] M. W. Lin, Q. S. Zhan, Z. S. Xu, and R. Q. Chen, "Group decisionmaking model with hesitant multiplicative preference relations based on regression method and feedback mechanism," IEEE Access, vol. 6, pp. 61130-61150, 2018.

[29] Z. M. Zhang and W. Pedrycz, "Iterative algorithms to manage the consistency and consensus for group decision-making with hesitant multiplicative preference relations," IEEE Trans. Fuzzy Syst., vol. 28, no. 11, pp. 2944-2957, Nov. 2020.

[30] Y. J. Xu, M. Q. Li, F. J. Cabrerizo, F. Chiclana, and E. HerreraViedma, "Algorithms to detect and rectify multiplicative and ordinal inconsistencies of fuzzy preference relations," IEEE Trans. Syst., Man, Cybern., Syst., vol. 51, no. 6, pp. 3498-3511, Jun. 2021, doi: 10.1109/TSMC.2019.2931536.

[31] Y. J. Xu and F. Herrera, "Visualizing and rectifying different inconsistencies for fuzzy reciprocal preference relations," Fuzzy Sets Syst., vol. 362, pp. 85-109, May 2019.

[32] Y. J. Xu, J. N. D. Gupta, and H. M. Wang, "The ordinal consistency of an incomplete reciprocal preference relation," Fuzzy Sets Syst., vol. 246, no. 4, pp. 62-77, 2014.
[33] F. Liu, Y.-N. Peng, Q. Yu, and H. Zhao, "A decision-making model based on interval additive reciprocal matrices with additive approximationconsistency," Inf. Sci., vol. 422, pp. 161-176, Jan. 2018.

[34] F. Liu, "Acceptable consistency analysis of interval reciprocal comparison matrices," Fuzzy Sets Syst., vol. 160, pp. 2686-2700, Sep. 2009.

[35] Z.-J. Wang and K. W. Li, "Goal programming approaches to deriving interval weights based on interval fuzzy preference relations," Inf. Sci., vol. 193, pp. 180-198, Jun. 2012.

[36] F. Y. Meng, J. Lin, C. Q. Tan, and Q. Zhang, "A new multiplicative consistency based method for decision making with triangular fuzzy reciprocal preference relations," Fuzzy Sets Syst., vol. 315, pp. 1-25, May 2017.

[37] Z. Zhang, X. Y. Kou, and Q. X. Dong, "Additive consistency analysis and improvement for hesitant fuzzy preference relations," Expert Syst. Appl., vol. 98, pp. 118-128, May 2018.

[38] J. A. Morente-Molinera, G. Kou, C. Pang, F. J. Cabrerizo, and E. Herrera-Viedma, "An automatic procedure to create fuzzy ontologies from users' opinions using sentiment analysis procedures and multi-granular fuzzy linguistic modelling methods," Inf. Sci., vol. 476, pp. 222-238, Feb. 2019.

[39] C.-C. Li, Y. C. Dong, Y. J. Xu, F. Chiclana, E. Herrera-Viedma, and F. Herrera, "An overview on managing additive consistency of reciprocal preference relations for consistency-driven decision making and fusion: Taxonomy and future directions," Inf. Fusion, vol. 52, pp. 143-156, Dec. 2019.

[40] Y. J. Xu, X. Liu, and H. M. Wang, "The additive consistency measure of fuzzy reciprocal preference relations," Int. J. Mach. Learn. Cybern., vol. 9, no. 7, pp. 1141-1152, 2017.

[41] Z. Zhang and C. H. Guo, "Fusion of heterogeneous incomplete hesitant preference relations in group decision making," Int. J. Comput. Intell. Syst., vol. 9, no. 2, pp. 245-262, 2016.

[42] Y. Lin, Y.-M. Wang, and S.-Q. Chen, "Multistage decision making based on prioritization of hesitant multiplicative preference relations," J. Intell. Fuzzy Syst., vol. 32, pp. 691-701, Jan. 2017.

[43] X. He and Y. Wu, "City sustainable development evaluation based on hesitant multiplicative fuzzy information," Math. Problems Eng., vol. 2017, no. 1, 2017, Art. no. 8306508.

[44] P. T. Harker, "Incomplete pairwise comparisons in the analytic hierarchy process," Math. Model., vol. 9, no. 11, pp. 837-848, 1987.

[45] Z. S. Xu, "A consensus reaching process under incomplete multiplicative preference relations," Int. J. Gen. Syst., vol. 41, no. 4, pp. 333-351, 2012.

[46] Y. J. Xu, C. Y. Li, and X. W. Wen, "Missing values estimation and consensus building for incomplete hesitant fuzzy preference relations with multiplicative consistency," Int. J. Comput. Intell. Syst., vol. 11, no. 1, pp. 101-119, 2018.

[47] T. L. Saaty and L. G. Vargas, "Uncertainty and rank order in the analytic hierarchy process," Eur. J. Oper. Res., vol. 32, no. 1, pp. 107-117, 1987.

[48] T. L. Saaty and L. G. Vargas, "Comparison of Eigenvalue, logarithmic least squares and least squares methods in estimating ratios," Math. Model., vol. 5, no. 5, pp. 309-324, 1984.

[49] T. L. Saaty, "Eigenvector and logarithmic least squares," Eur. J. Oper. Res., vol. 48, no. 1, pp. 156-160, 1990.

[50] Y.-M. Wang, J.-B. Yang, and D.-L. Xu, "A two-stage logarithmic goal programming method for generating weights from interval comparison matrices," Fuzzy Sets Syst., vol. 152, no. 3, pp. 475-498, 2005.

[51] Z. S. Xu and C. P. Wei, "A consistency improving method in the analytic hierarchy process," Eur. J. Oper. Res., vol. 116, pp. 443-449, Jul. 1999.

[52] K. W. Li, Z. J. Wang, and X. Y. Tong, "Acceptability analysis and priority weight elicitation for interval multiplicative comparison matrices," Eur. J. Oper. Res., vol. 250, pp. 628-638, Apr. 2016.

[53] Z. M. Zhang and S.-M. Chen, "Group decision making based on acceptable multiplicative consistency and consensus of hesitant fuzzy linguistic preference relations," Inf. Sci., vol. 541, pp. 531-550, Dec. 2020.

[54] J. Tang, Y. N. Zhang, H. Fujita, X. D. Zhang, and F. Y. Meng, "Analysis of acceptable additive consistency and consensus of group decision making with interval-valued hesitant fuzzy preference relations," Neural Comput. Appl., vol. 33, pp. 7747-7772, Jan. 2021, doi: 10.1007/s00521-020-05516-z.

[55] X. Liu, Y. J. Xu, R. Montes, R.-X. Ding, and F. Herrera, "Alternative ranking-based clustering and reliability index-based consensus reaching process for hesitant fuzzy large scale group decision making," IEEE Trans. Fuzzy Syst., vol. 27, no. 1, pp. 159-171, Jan. 2019.

[56] X. Liu, Y. J. Xu, and F. Herrera, "Consensus model for large-scale group decision making based on fuzzy preference relation with self-confidence: Detecting and managing overconfidence behaviors," Inf. Fusion, vol. 52, pp. 245-256, Dec. 2019. 
[57] M. J. del Moral, F. Chiclana, J. M. Tapia, and E. Herrera-Viedma, "A comparative study on consensus measures in group decision making," Int. J. Intell. Syst., vol. 33, no. 8, pp. 1624-1638, 2018.

[58] H. J. Zhang, Y. C. Dong, and X. Chen, "The 2-rank consensus reaching model in the multigranular linguistic multiple-attribute group decisionmaking," IEEE Trans. Syst., Man, Cybern., Syst., vol. 48, no. 12, pp. 2080-2094, Dec. 2018.

[59] Q. B. Zha, Y. C. Dong, H. J. Zhang, F. Chiclana, and E. Herrera-Viedma, "A personalized feedback mechanism based on bounded confidence learning to support consensus reaching in group decision making," IEEE Trans. Syst., Man, Cybern., Syst., vol. 51, no. 6, pp. 3900-3910, Jun. 2021, doi: 10.1109/TSMC.2019.2945922.

[60] H. Y. Wu, P. J. Ren, and Z. S. Xu, "Addressing site selection for earthquake shelters with hesitant multiplicative linguistic preference relation," Inf. Sci., vol. 516, pp. 370-387, Apr. 2020.

[61] H. H. Zhang, Y. Peng, H. Lin, G. D. Tian, and Z. W. Li, "A hybrid multi-objective optimization approach for energy-absorbing structures in train collisions," Inf. Sci., vol. 481, pp. 491-506, May 2019.

[62] G. D. Tian et al., "Fuzzy grey choquet integral for evaluation of multicriteria decision making problems with interactive and qualitative indices,' IEEE Trans. Syst., Man, Cybern., Syst., vol. 51, no. 3, pp. 1855-1868, Mar. 2021.

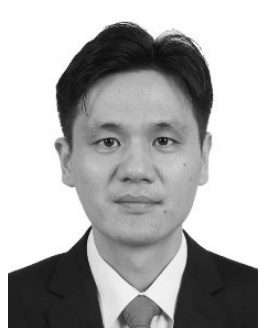

Yejun Xu received the M.S. and Ph.D. degrees in management science and engineering from Southeast University, Nanjing, China, in 2005 and 2009, respectively.

$\mathrm{He}$ is currently a Professor with the College of Management and Economics, Tianjin University, Tianjin, China. He has authored and coauthored more than 120 articles to professional journals, such as IEEE TRANSACTIONS ON SYSTEMS, MAN, AND CybeRnETICS: Systems, IEEE TRANSACTIONS ON FuZZY Systems, Fuzzy Sets and Systems, Information Sciences, International Journal of Approximate Reasoning, and Knowledge-Based Systems. His current research interests include group decision making under uncertainty and conflict analysis.

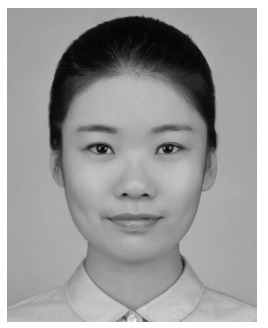

Mengqi $\mathbf{~ L i}$ is currently pursuing the $\mathrm{Ph} . \mathrm{D}$. degree in management science and engineering with the Business School, Hohai University, Nanjing, China.

Her current research interests include decisionmaking analysis, consensus reaching process, hesitant fuzzy set, and large-scale decision making.

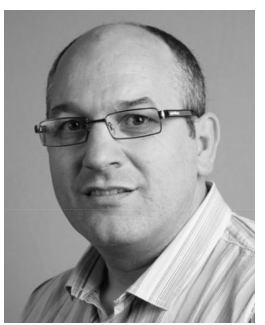

Francisco Chiclana received the B.Sc. and Ph.D. degrees in mathematics from the University of Granada, Granada, Spain, in 1989 and 2000, respectively.

$\mathrm{He}$ is a Professor of Computational Intelligence and Decision Making with the School of Computer Science and Informatics, Faculty of Computing, Engineering, and Media, De Montfort University, Leicester, U.K. From 2015 to 2018, he was an Honorary Professor with the Department of Mathematics, University of Leicester, Leicester, and currently a Visiting Scholar with the Department of Computer Science and Artificial Intelligence, University of Granada. His research areas as fuzzy preference modeling, decision support systems, consensus, recommender systems, social networks, rationality/consistency, and aggregation.

Prof. Chiclana is an associate editor and a guest editor for several ISI indexed journals. He has organized and chaired special sessions/workshops in many major international conferences. Clarivate Analytics has currently classed him as a Highly Cited Researcher in Computer Sciences.

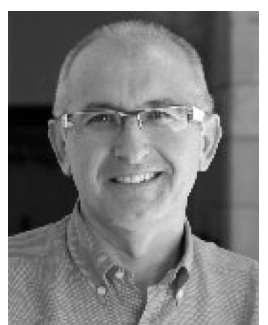

Enrique Herrera-Viedma (Fellow, IEEE) received the M.Sc. and Ph.D. degrees in computer science from the University of Granada, Granada, Spain, in 1993 and 1996, respectively.

$\mathrm{He}$ is a Professor of Computer Science and AI with the Andalusian Research Institute in Data Science and Computational Intelligence and the Vice-President of Research and Knowledge Transfer with the University of Granada. He is also a Research Fellow with Universiti Teknologi Malaysia, Johor Bahru, Malaysia. His H-index is 85 with more than 24000 citations received in Web of Science and 102 in Google Scholar with more than 42000 cites received. He has been identified as Highly Cited Researcher by Clarivate Analytics in both scientific categories, Computer Science and Engineering, from 2014 to 2020. His current research interests include group decision making, consensus models, linguistic modeling, aggregation of information, information retrieval, bibliometric, digital libraries, Web quality evaluation, recommender systems, and social media.

Prof. Herrera-Viedma is a Vice-President for Cybernetics in the prestigious IEEE SMC Society and an Associate Editor in several journals, such as IEEE TRANSACTIONS ON FUZZY SYSTEMS, IEEE TRANSACTIONS ON SYSTEMS, Man, And Cybernetics: Systems, Information Sciences, Applied Soft Computing, and Knowledge-Based Systems. 